\title{
AN EQUIVARIANT WHITEHEAD ALGORITHM AND CONJUGACY FOR ROOTS OF DEHN TWIST AUTOMORPHISMS
}

\author{
SAVA KRSTIĆ ${ }^{1}$, MARTIN LUSTIG $^{2}$ AND KAREN VOGTMANN ${ }^{3}$ \\ ${ }^{1}$ Department of Computer Science and Engineering, Oregon Graduate Institute, \\ PO Box 91000, Portland, OR 97291, USA (krstic@cse.ogi.edu) \\ ${ }^{2}$ Laboratoire de Mathématiques, Université d'Aix-Marseille III, Ave. E. \\ Normandie-Niemen, 13397 Marseille 20, France (Martin.Lustig@math.u-3mrs.fr) \\ ${ }^{3}$ Department of Mathematics, 555 Malott Hall, Cornell University, \\ Ithaca, NY 14853, USA (vogtmann@math.cornell.edu)
}

(Received 11 January 1999)

\begin{abstract}
Given finite sets of cyclic words $\left\{u_{1}, \ldots, u_{k}\right\}$ and $\left\{v_{1}, \ldots, v_{k}\right\}$ in a finitely generated free group $F$ and two finite groups $A$ and $B$ of outer automorphisms of $F$, we produce an algorithm to decide whether there is an automorphism which conjugates $A$ to $B$ and takes $u_{i}$ to $v_{i}$ for each $i$. If $A$ and $B$ are trivial, this is the classic algorithm due to Whitehead. We use this algorithm together with Cohen and Lustig's solution to the conjugacy problem for Dehn twist automorphisms of $F$ to solve the conjugacy problem for outer automorphisms which have a power which is a Dehn twist. This settles the conjugacy problem for all automorphisms of $F$ which have linear growth.
\end{abstract}

Keywords: automorphisms of free groups; conjugacy problem; Dehn twists; Whitehead algorithm

AMS 2000 Mathematics subject classification: Primary 20F32

Secondary 57M07

\section{Introduction}

In 1912, Max Dehn formulated fundamental problems concerning a group given by generators and relations. One of these, the conjugacy problem, asks whether there is an algorithm to decide whether two words in the generators represent conjugate elements of the group. Dehn himself gave an elegant solution to this problem in the case when the group is the fundamental group of a closed hyperbolic surface, given by the standard presentation.

For the group $\operatorname{Out}\left(F_{n}\right)$ of outer automorphisms of a free group of rank $n$, the conjugacy problem has been solved for various classes of elements. For finite-order elements of $\operatorname{Out}\left(F_{n}\right)$, and in fact for finite groups of automorphisms, an algorithm to solve the conjugacy problem follows from results of Krstić [8]. Both Los [11] and Sela [14] have published solutions to the conjugacy problem for automorphisms which are irreducible in the sense of Bestvina and Handel [2]. Finally, the conjugacy problem has been solved 
by Cohen and Lustig [5] for Dehn twist automorphisms, which are outer automorphisms given in terms of a graph-of-groups decomposition of $F_{n}$ and which include the automorphisms induced by Dehn twists of surfaces.

Our first theorem solves the conjugacy problem for finite groups of automorphisms, under the additional constraint that the conjugating automorphism must take one given finite set of words to another. We give an algorithm, called the equivariant Whitehead algorithm, and prove the following.

Theorem 1.1. Given homomorphisms $\alpha$ and $\beta$ from a finite group $G$ to $\operatorname{Out}\left(F_{n}\right)$ and cyclic words $u_{1}, \ldots, u_{k}, v_{1}, \ldots, v_{k}$ in $F_{n}$, the equivariant Whitehead algorithm decides whether there is an outer automorphism $\phi$ of $F_{n}$ such that $\phi^{-1} \alpha(g) \phi=\beta(g)$ for all $g \in G$ and $\phi\left(u_{i}\right)=v_{i}$ for all $i$. Furthermore, the algorithm finds $\phi$ if it exists.

When $G=1$, our algorithm is the classical algorithm of Whitehead [15].

In our second main theorem, we apply the equivariant Whitehead algorithm, in the special case where $G$ is a cyclic group, to solve the conjugacy problem for automorphisms with powers which are Dehn twist automorphisms.

Theorem 1.2. Given two outer automorphisms $\phi_{1}$ and $\phi_{2}$ of $F_{n}$ which each have powers that are Dehn twist automorphisms, Algorithm 5.8 decides whether or not there exists an outer automorphism $\psi$ of $F_{n}$ that conjugates $\phi_{1}$ to $\phi_{2}$. Furthermore, the algorithm finds $\psi$ if it exists.

A natural invariant of the conjugacy class of an outer automorphisms $\phi$ is its growth rate, where $\phi$ is said to have polynomial growth of degree $d$ (respectively, exponential growth) if for each cyclic word $w$ the length of $\phi^{k}(w)$ is bounded above (respectively, below) by a degree- $d$ polynomial (respectively, exponential) function of $k$. Finite-order automorphisms have constant growth, infinite order irreducible automorphisms grow exponentially, and Dehn twist automorphisms have linear growth. Note that an automorphism has the same growth rate as any finite power of the automorphism.

It is a common occurrence in the study of automorphisms of free groups that one must pass to a finite power to get an automorphism into a standard form (see, for example, $[\mathbf{3}]$ ). Using train-track techniques, it is not hard to show that any automorphism of linear growth has a power that is a Dehn twist automorphism. Thus, Theorem 1.2 completes the solution of the conjugacy problem for linear growth automorphisms. Lustig $[\mathbf{1 2}, \mathbf{1 3}]$ has given a complete solution to the conjugacy problem for $\operatorname{Out}\left(F_{n}\right)$, which relies in a fundamental way on the results of this paper.

The paper is organized as follows. In $\S 2$ we translate the algebraic problem solved by the equivariant Whitehead algorithm into a geometric problem, and show how to derive the solution using an equivariant version of a technical process known as peak reduction.

In $\S 3$ we prove the necessary equivariant version of the classical peak reduction lemma. Equivariant peak reduction lemmas have previously been proved in Kalajdžievski $[\mathbf{7}]$ and Krstić [9]. Unfortunately, however, the result we need cannot easily be derived from these, so we present a complete proof here that is somewhat simpler and is suited to our needs.

In $\S 4$ we review the definition of a Dehn twist automorphism and prove some basic lemmas. In $\S 5$ we use the equivariant Whitehead algorithm to solve the conjugacy problem 


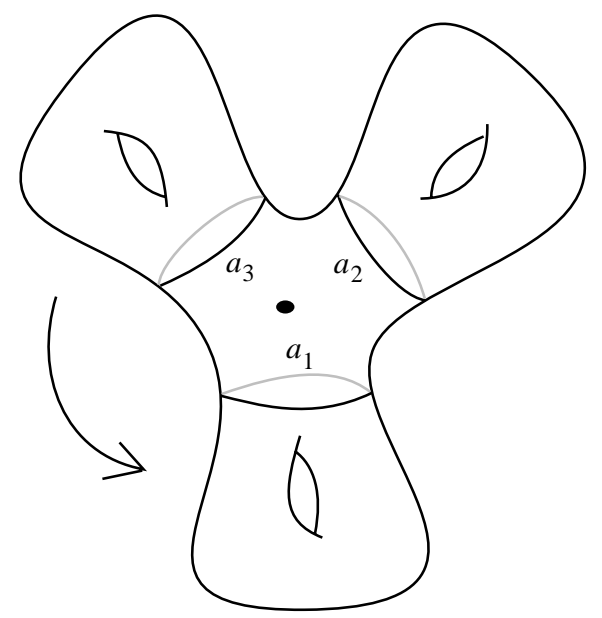

Figure 1. Example 1.3.

for roots of Dehn twist automorphisms. The general idea of the solution is the following. The main structure theorem of $[4]$ associates to a given Dehn twist automorphism $\hat{D}$ a decomposition of $F_{n}$ as a graph of groups $\mathcal{G}$ which is preserved by $\hat{D}$. Since a root $\phi$ of $\hat{D}$ commutes with $\hat{D}$, it follows that $\phi$ too must preserve the graph-of-groups structure $\mathcal{G}$. The Dehn twist automorphism $\hat{D}$ leaves each of the vertex and edge groups of $\mathcal{G}$ invariant and induces the identity on these groups up to inner automorphism, but the root $\phi$ may permute the vertex groups. Even if $\phi$ sends a vertex group $G_{v}$ to itself, it may induce a non-trivial finite-order outer automorphism of $G_{v}$, in which case $\phi$ permutes the conjugacy classes in $G_{v}$ of the twistors associated to edges adjacent to $v$. In Theorem 5.7, we show that up to combinatorial data that are easily derived from the graph of groups, two roots of Dehn twist automorphisms are conjugate if and only if these finite-order outer automorphisms of the vertex groups are conjugate by an automorphism that takes the twistor conjugacy classes of one to the those of the other. The equivariant Whitehead algorithm is then applied to decide whether such a conjugating automorphism exists.

The following example may help illuminate the issues involved in deciding whether roots of Dehn twist automorphisms are conjugate.

Example 1.3. Consider a 2-sphere that has one puncture and three handles attached, symmetrically arranged around the puncture (see Figure 1). Its fundamental group is a free group of rank 6 . Let $\alpha_{1}, \alpha_{2}$ and $\alpha_{3}$ be symmetrically arranged disjoint curves, such that $\alpha_{i}$ separates the $i$-th handle from the rest of the surface.

Define homeomorphisms $h_{1}$ and $h_{2}$ of the surface as follows. For $h_{1}$, rotate the surface by an angle of $2 \pi / 3$ around an axis that goes through the puncture (thereby permuting the handles cyclically), and then twist once around each $\alpha_{i}$. For $h_{2}$, do the same rotation, then twist three times around $\alpha_{1}$ but do not twist around $\alpha_{2}$ or $\alpha_{3}$. Then $h_{1}$ and $h_{2}$ are (third) roots of Dehn twists. Let $\phi_{1}$ and $\phi_{2}$ be the automorphisms of the fundamental group induced by $h_{1}$ and $h_{2}$, respectively.

Question: Are $\phi_{1}$ and $\phi_{2}$ conjugate in $\operatorname{Out}\left(F_{n}\right)$ ? 
In $\S 5$ we translate this example into the language of graphs of groups and answer it.

\section{Equivariant Whitehead algorithm}

We model automorphisms of a free group by homotopy equivalences of graphs. In this section we translate the necessary ideas into graph-theoretical terms, state the equivariant peak reduction theorem, and show how it is used to obtain the equivariant Whitehead algorithm.

\subsection{Marked $G$-graphs}

By a graph $\Gamma$ we mean a finite connected one-dimensional CW complex. Maps between graphs are assumed to send vertices to vertices. When convenient, we think of $\Gamma$ as a metric space by assigning all edges length one.

We identify $F_{n}$ with $\pi_{1}\left(R_{n}, v_{0}\right)$, where $R_{n}$ is the graph with one vertex $v_{0}$ and $n$ edges. Any automorphism $\phi$ of $F_{n}$ can be represented by a homotopy equivalence $R_{n} \rightarrow R_{n}$. There is a unique homotopy equivalence representing $\phi$ which is locally injective, linear on edges, and stretches each edge uniformly; we will abuse notation and call this map $\phi$ also.

If $G$ is a finite group, a $G$-graph is a graph $\Gamma$ together with an action of $G$ on $\Gamma$ by isometries. A $G$-graph is reduced if there are no $G$-invariant forests in $\Gamma$. Any forest $E$ in $\Gamma$ determines an equivalence relation on points of $\Gamma$, with $x \sim y$ if $x$ and $y$ belong to the same connected component of $E$. The quotient map $\Gamma \rightarrow \Gamma / \sim$ is called a forest collapse. If the forest is $G$-invariant, the action of $G$ on $\Gamma$ induces an action on the quotient $\Gamma / \sim$.

A marking on $\Gamma$ is a homotopy equivalence $h: R_{n} \rightarrow \Gamma$. A marking gives an isomorphism of $F_{n}$ with the fundamental group of $\Gamma$. To any $G$-graph $\Gamma$ and marking $h$, we associate a homomorphism $\alpha: G \rightarrow \operatorname{Out}\left(F_{n}\right)$, defined by $\alpha(g)=\left(h^{-1} g_{\Gamma} h\right)_{*}$, where $h^{-1}$ is a homotopy inverse to $h$, and $g_{\Gamma}$ is the isometry of $\Gamma$ corresponding to $g$. We say that the pair $(\Gamma, h)$ realizes $\alpha$.

By the Realization Theorem (see $[\mathbf{6}]$ or $[\mathbf{1 6}]$ ), every homomorphism $\alpha: G \rightarrow \operatorname{Out}\left(F_{n}\right)$ is realized by some marked $G$-graph $(\Gamma, h)$. By collapsing a maximal $G$-invariant forest in $\Gamma$, we obtain a marked, reduced $G$-graph that also realizes $\alpha$.

\subsection{Whitehead moves}

Two reduced $G$-graphs $\Gamma_{1}$ and $\Gamma_{2}$ are said to be connected by a Whitehead move if there exists a (non-reduced) $G$-graph $\Gamma$ and edges $e_{1}$ and $e_{2}$ in $\Gamma$ whose orbits $G e_{1}$ and $G e_{2}$ are forests that collapse to give $\Gamma_{1}$ and $\Gamma_{2}$, respectively,

$$
\Gamma_{1} \stackrel{c_{1}}{\longleftarrow} \Gamma \stackrel{c_{2}}{\longrightarrow} \Gamma_{2} .
$$

We can construct an explicit $G$-equivariant homotopy inverse $f_{i}: \Gamma_{i} \rightarrow \Gamma$ by 'folding together' initial segments of appropriate edges at vertices of $\Gamma_{i}$, as follows. Let $E=G e_{i}$ be the edge orbit in $\Gamma$ which is collapsed to give $\Gamma_{i}$. If $x_{i}, y_{i} \in \Gamma_{i}$ are not in the image $c_{i}(E)$, then they correspond to unique points $x, y$ in $\Gamma$. In this case, say $x_{i} \sim y_{i}$ if $d(x, v)=d(y, v) \leqslant 1 / 3$ for some extremal vertex $v$ of the forest $E$. The map $f_{i}$ is the 
quotient map $\Gamma_{i} \rightarrow \Gamma_{i} / \sim$ followed by a rescaling so that all edges have length one. The graph $\Gamma_{i} / \sim$ is naturally homeomorphic to $\Gamma$, and the induced action of $G$ on $\Gamma_{i}$ is the original action on $\Gamma$.

The composition $w=c_{2} f_{1}: \Gamma_{1} \rightarrow \Gamma_{2}$ is a $G$-equivariant homotopy equivalence, which we will also call a Whitehead move. A composition of Whitehead moves will be called an equivariant Whitehead path. If $w: \Gamma_{1} \rightarrow \Gamma_{2}$ is an equivariant Whitehead path, $h_{1}: R_{n} \rightarrow$ $\Gamma_{1}$ is a marking realizing $\alpha: G \rightarrow \operatorname{Out}\left(F_{n}\right)$, and $h_{2}: R_{n} \rightarrow \Gamma_{2}$ realizes $\beta: G \rightarrow \operatorname{Out}\left(F_{n}\right)$, then $\phi=\left(h_{2}^{-1} w h_{1}\right)_{*}$ conjugates $\alpha$ to $\beta$, i.e. $\phi \alpha(g) \phi^{-1}=\beta(g)$ for all $g \in G$.

We have the following converse statement.

Theorem 2.1. Let $\alpha$ and $\beta$ be two homomorphisms from $G$ to $\operatorname{Out}\left(F_{n}\right)$, realized by reduced marked $G$-graphs $\left(\Gamma_{1}, h_{1}\right)$ and $\left(\Gamma_{2}, h_{2}\right)$, respectively, and let $\phi$ be an outer automorphism of $F_{n}$ with $\phi \alpha(g) \phi^{-1}=\beta(g)$ for all $g \in G$. Then there is an equivariant Whitehead path $w: \Gamma_{1} \rightarrow \Gamma_{2}$ with $\phi=\left(h_{2}^{-1} w h_{1}\right)_{*}$.

Proof. Corollary 1 of $[\mathbf{8}]$ implies that every equivariant isomorphism $\pi_{1}(\Gamma) \rightarrow \pi_{1}\left(\Gamma^{\prime}\right)$ between reduced $G$-graphs is induced by an equivariant homotopy equivalence $\Gamma \rightarrow$ $\Gamma^{\prime}$. By Proposition 4 of [8], every equivariant homotopy equivalence $\Gamma_{1} \rightarrow \Gamma_{2}$ between reduced $G$-graphs is a product of Whitehead moves.

\subsection{Equivariant peak reduction}

If $\Gamma$ is a reduced $G$-graph and $l$ is a homotopy class of loops in $\Gamma$, we define $|l|_{\Gamma}$ to be equal to the edge-path length of the cyclically reduced edge-path loop in $\Gamma$ homotopic to $l$. If $L=\left\{l_{1}, \ldots, l_{k}\right\}$ is a set of homotopy classes of loops in $\Gamma$, define $|L|_{\Gamma}$ to be the sum of the $\left|l_{i}\right|_{\Gamma}$, for $l_{i} \in L$.

Theorem 2.2 (equivariant peak reduction). Let $G$ be a finite group, let $\Gamma$ be a reduced $G$-graph, and let $L$ be a finite invariant set of homotopy classes of loops in $\Gamma$. Let $w^{\prime}: \Gamma \rightarrow \Gamma^{\prime}$ and $w^{\prime \prime}: \Gamma \rightarrow \Gamma^{\prime \prime}$ be equivariant Whitehead moves, with

$$
\left|w^{\prime} L\right|_{\Gamma^{\prime}} \leqslant|L|_{\Gamma}>\left|w^{\prime \prime} L\right|_{\Gamma^{\prime \prime}}
$$

Then there is a sequence $w_{i}: \Gamma_{i-1} \rightarrow \Gamma_{i}, 1 \leqslant i \leqslant m$, of equivariant Whitehead moves from $\Gamma^{\prime}=\Gamma_{0}$ to $\Gamma^{\prime \prime}=\Gamma_{m}$ with $w_{m} \ldots w_{1} w^{\prime} L=w^{\prime \prime} L$ and $\left|w_{i} \ldots w_{1} w^{\prime} L\right|_{\Gamma_{i}}<|L|_{\Gamma}$ for all $i, 1 \leqslant i \leqslant m$.

The proof of this theorem will be given in the next section.

Corollary 2.3. If $w: \Gamma^{\prime} \rightarrow \Gamma^{\prime \prime}$ is a $G$-equivariant Whitehead path, and $L$ a finite set of homotopy classes of loops in $\Gamma^{\prime}$, then there is a sequence $w_{i}: \Gamma_{i-1} \rightarrow \Gamma_{i}, 1 \leqslant i \leqslant r$, of equivariant Whitehead moves from $\Gamma^{\prime}=\Gamma_{0}$ to $\Gamma^{\prime \prime}=\Gamma_{r}$ with $w_{r} \ldots w_{1} L=w L$ and $\left|w_{i} \ldots w_{1} L\right|_{\Gamma_{i}} \leqslant \max \left(|L|_{\Gamma^{\prime}},|w L|_{\Gamma^{\prime \prime}}\right)$ for all $0 \leqslant i \leqslant r$.

Proof. Let $M$ be the maximum length of the image $w_{0} L$ under any initial segment $w_{0}$ of the Whitehead path $w$. If $M>\max \left(|L|_{\Gamma^{\prime}},|w L|_{\Gamma^{\prime \prime}}\right)$, we can find a reduced $G$-graph $\Gamma$ on the path with $\left|w_{0} L\right|_{\Gamma}=M$ to which we can apply Theorem 2.2. This reduces the number of reduced $G$-graphs on the path realizing the maximum $M$. 


\subsection{Equivariant Whitehead algorithm}

We are now ready to describe the equivariant Whitehead algorithm. We have a finite group $G$, two homomorphisms $\alpha, \beta: G \rightarrow \operatorname{Out}\left(F_{n}\right)$, and sets of cyclic words $U=$ $\left\{u_{1}, \ldots, u_{k}\right\}$ and $V=\left\{v_{1}, \ldots, v_{k}\right\}$ in $F_{n}$. We want to decide whether there is an automorphism $\phi$ which conjugates $\alpha$ to $\beta$ and takes $u_{i}$ to $v_{i}$ for all $i$. Furthermore, if $\phi$ exists, we want the algorithm to find $\phi$. If such a $\phi$ exists, its effect on $\alpha(g) u_{i}$ is determined by its effect on $u_{i}$, so, without loss of generality, we may assume that $U$ and $V$ are invariant under the action of $G$.

We first find reduced marked $G$-graphs $\left(\Gamma_{\alpha}, h_{\alpha}\right)$ realizing $\alpha$ and $\left(\Gamma_{\beta}, h_{\beta}\right)$ realizing $\beta$. This can be done by the Realization Theorem. The folding algorithm of Bestvina and Handel $[\mathbf{2}]$ finds $\left(\Gamma_{\alpha}, h_{\alpha}\right)$ and $\left(\Gamma_{\beta}, h_{\beta}\right)$ efficiently.

Let $m=\max \left\{\left|h_{\alpha}(U)\right|_{\Gamma_{\alpha}},\left|h_{\beta}(V)\right|_{\Gamma_{\beta}}\right\}$. We construct a graph $\Delta=\Delta(G, k, m)$. The vertices of $\Delta$ are all pairs $(\Gamma, L)$, where $\Gamma$ is a reduced $G$-graph and $L=\left(l_{1}, \ldots, l_{k}\right)$ is an ordered, $G$-invariant set of homotopy classes of loops, whose reduced edge-path representatives have total length at most $m$. Two vertices $\left(\Gamma^{\prime}, L^{\prime}\right)$ and $\left(\Gamma^{\prime \prime}, L^{\prime \prime}\right)$ are connected if there is an equivariant Whitehead move $w: \Gamma^{\prime} \rightarrow \Gamma^{\prime \prime}$ with $w\left(l_{i}^{\prime}\right)=l_{i}^{\prime \prime}$ for all $i$. Note that $\Delta$ is a finite graph: there are only finitely many reduced $G$-graphs with fundamental group isomorphic to $F_{n}$, there are only finitely many ordered $k$-tuples of reduced edge-path loops with a given total length, and there are only finitely many equivariant Whitehead moves starting at any reduced $G$-graph.

Theorem 2.4. In the notation above, an automorphism $\phi$ conjugating $\alpha$ to $\beta$ and sending $u_{i}$ to $v_{i}$ exists if and only if the pairs $\left(\Gamma_{\alpha}, h_{\alpha}(U)\right)$ and $\left(\Gamma_{\beta}, h_{\beta}(V)\right)$ are connected in the graph $\Delta(G, k, m)$.

Proof. If there is a $G$-equivariant Whitehead path $w$ from $\Gamma_{\alpha}$ to $\Gamma_{\beta}$, taking $h_{\alpha}\left(u_{i}\right)$ to $h_{\beta}\left(v_{i}\right)$ for all $i$, then $\phi=\left(h_{\beta}^{-1} w h_{\alpha}\right)_{*}$ conjugates $\alpha$ to $\beta$, and $w h_{\alpha}\left(u_{i}\right)$ is homotopic to $h_{\beta}\left(v_{i}\right)$, so $\phi\left(u_{i}\right)=v_{i}$.

Conversely, suppose there is an automorphism $\phi$ with $\phi \alpha(g) \phi^{-1}=\beta(g)$ for all $g \in G$ and $\phi\left(u_{i}\right)=v_{i}$ for all $i$. By Theorem 2.1, there is a Whitehead path $w: \Gamma_{\alpha} \rightarrow \Gamma_{\beta}$ with $\phi=\left(h_{\beta}^{-1} w h_{\alpha}\right)_{*}$. Since $\left(h_{\beta}^{-1} w h_{\alpha}\right)_{*}\left(u_{i}\right)=v_{i}$, we have $w h_{\alpha}\left(u_{i}\right)$ homotopic to $h_{\beta}\left(v_{i}\right)$. By Corollary 2.3, we can find a new Whitehead path $w_{r} \ldots w_{1}$ with $w_{r} \ldots w_{1} h_{\alpha}\left(u_{i}\right)=h_{\beta}\left(v_{i}\right)$ and

$$
\left|w_{j} \ldots w_{1} h_{\alpha}(U)\right| \leqslant \max \left\{\left|h_{\alpha}(U)\right|_{\Gamma_{\alpha}},\left|h_{\beta}(V)\right|_{\Gamma_{\beta}}\right\},
$$

for all initial segments $w_{j} \ldots w_{1}$ of the path. This path is in $\Delta(G, k, m)$.

Remark 2.5. The graph $\Delta(G, k, m)$ is very large. A more practical algorithm can be obtained by observing the following corollary of Theorem 2.2 .

Corollary 2.6. Let $(\Gamma, h)$ be a marked $G$-graph realizing $\alpha$, and suppose there is another marked $G$-graph $\left(\Gamma^{\prime}, h^{\prime}\right)$ realizing $\alpha$ with $\left|h^{\prime}(U)\right|_{\Gamma^{\prime}}<|h(U)|_{\Gamma}$. Then there is a Whitehead move $w: \Gamma \rightarrow \Gamma_{1}$ that reduces $|h(U)|$.

Using this corollary we can find $\left(\Gamma_{\alpha}, h_{\alpha}\right)$ and $\left(\Gamma_{\beta}, h_{\beta}\right)$ realizing $\alpha$ and $\beta$ with $\left|h_{\alpha}(U)\right|$ and $\left|h_{\beta}(V)\right|$ minimal. 
If $\phi$ exists, then the marked $G$-graph $\left(\Gamma_{\beta}, h_{\beta} \phi\right)$ realizes $\alpha$, so

$$
\left|h_{\beta}(V)\right|_{\Gamma_{\beta}}=\left|h_{\beta} \phi(U)\right|_{\Gamma_{\beta}} \geqslant\left|h_{\alpha}(U)\right|_{\Gamma_{\alpha}} .
$$

Similarly, $\left(\Gamma_{\alpha}, h_{\alpha} \phi^{-1}\right)$ realizes $\beta$, so $\left|h_{\alpha}(U)\right|_{\Gamma_{\alpha}} \geqslant\left|h_{\beta}(V)\right|_{\Gamma_{\beta}}$, giving

$$
\left|h_{\alpha}(U)\right|_{\Gamma_{\alpha}}=\left|h_{\beta} \phi(U)\right|_{\Gamma_{\beta}}=\left|h_{\beta}(V)\right|_{\Gamma_{\beta}} .
$$

If $\left|h_{\alpha}(U)\right|_{\Gamma_{\alpha}}=\left|h_{\beta} \phi(U)\right|_{\Gamma_{\beta}}=m_{0}$, we can apply Corollary 2.6 to find a $G$-equivariant Whitehead path $w: \Gamma_{u} \rightarrow \Gamma_{v}$ with $w h_{\alpha}(U)=h_{\beta} \phi(U)=h_{\beta}(V)$. By Corollary 2.3, we can then find a Whitehead path $w_{r} \ldots w_{1}$ with $w_{r} \ldots w_{1} h_{\alpha}(U)=h_{\beta} \phi(U)=h_{\beta}(V)$ and $\left|w_{j} \ldots w_{1} h_{\alpha}(U)\right|$ minimal for all $j$. Thus, to decide the existence of $\phi$, we need only check whether $\left(\Gamma_{\alpha}, h_{\alpha}(U)\right)$ and $\left(\Gamma_{\alpha}, h_{\beta}(V)\right)$ are connected in the graph $\Delta^{\prime}=\Delta^{\prime}\left(G, k, m_{0}\right)$, whose vertices are pairs $(\Gamma, L)$, where $\Gamma$ is a $G$-graph and $L$ is an ordered set of $k$ homotopy classes of loops, with total length equal to $m_{0}$. Two vertices of $\Delta^{\prime}$ are connected by an edge in the same way as those of $\Delta$.

\section{Proof of equivariant peak reduction lemma}

This section contains the proof of the equivariant peak reduction lemma. To prove this, we must understand what happens to the length of an edge-path under an equivariant Whitehead move $w$ that is the composition of an equivariant fold $f_{1}$ and collapse $c_{2}$ :

$$
\Gamma_{1} \stackrel{f_{1}}{\longrightarrow} \Gamma \stackrel{c_{2}}{\longrightarrow} \Gamma_{2}
$$

\subsection{Ideal edges and Whitehead moves}

We examine Whitehead moves from a slightly different point of view. The segments of edges that are identified by the fold $f_{1}$ to form a single new edge of $\Gamma$ can be thought of as a set of oriented edges in $\Gamma_{1}$; this set is called an ideal edge of $\Gamma_{1}$ if, given a marking $h: R_{n} \rightarrow \Gamma_{1}$, the reduced marked $G$-graph $\left(\Gamma_{2}, w h\right)$ is distinct from $\left(\Gamma_{1}, h\right)$. Conditions (1)-(4) below are necessary and sufficient for a set of oriented edges $\mathcal{I}$ of $\Gamma_{1}$ to be an ideal edge.

(1) All edges in $\mathcal{I}$ must terminate at a single vertex $v$.

This guarantees that we will be able to fold the edges in $\mathcal{I}$ together.

(2) For $x \in G, x(\mathcal{I}) \cap \mathcal{I}$ is either empty or all of $\mathcal{I}$.

This allows us to extend the action of $G$ to the equivariantly folded graph.

(3) $\mathcal{I}$ contains at least two, and at most all but two, of the edges at $v$.

This guarantees that we are not trivially adding a bivalent vertex.

(4) There is an element $a \in \mathcal{I}$ such that the intersection of $\mathcal{I}$ with the orbit of $a$ is the singleton $\{a\}$ and the intersection of $\mathcal{I}$ with the orbit of $a^{-1}$ is empty. 
This last condition guarantees that the graph $\left(\Gamma_{2}, w h\right)$ is different from $\left(\Gamma_{1}, h\right)$. To see this, note that we need $\Gamma$ to have a collapsible edge orbit different from the orbit of the new edge obtained by folding $\mathcal{I}$. Since $a$ is not in the orbit of the new edge, $a$ corresponds to an edge of $\Gamma_{1}$. The orbit of $a$ in $\Gamma_{1}$ is not a forest (since $\Gamma_{1}$ is reduced), but condition (4) guarantees that the orbit of $a$ in $\Gamma$ is a forest.

Let $P$ be the stabilizer of $\mathcal{I}$, i.e. the set of $x \in G$ with $x \mathcal{I}=\mathcal{I}$. One checks easily that for any $e \in \mathcal{I}, \operatorname{stab}(e) \leqslant P$, and for any $a$ satisfying condition $(4), \operatorname{stab}(a)=P$.

If $\mathcal{I}$ is an ideal edge, and $a \in \mathcal{I}$ satisfies condition (4), we denote the corresponding equivariant fold by $f_{\mathcal{I}}$, and the collapse of the edge orbit $G a$ by $c_{a}$; the Whitehead move $c_{a} \circ f_{\mathcal{I}}$ is denoted by $(\mathcal{I}, a)$, and the new reduced $G$-graph obtained from $\Gamma$ by $(\mathcal{I}, a) \Gamma$.

Two ideal edges $\mathcal{I}$ and $\mathcal{J}$ are compatible if either

(i) $\mathcal{I} \cap x \mathcal{J}=\emptyset$ for all $x \in G$; or

(ii) for some $x \in G$, we have $\mathcal{I} \subset x \mathcal{J}$ or $x \mathcal{J} \subset \mathcal{I}$.

Remark 3.1. The $G$-equivariant Nielsen moves of $[8]$ are the Whitehead moves of the form $(G e, a)$; this can be thought of as equivariantly 'sliding $e$ across $a$ '.

\subsection{The star graph}

We now consider the effect of a Whitehead move on the lengths of a set $L=\left\{l_{1}, \ldots, l_{k}\right\}$ of homotopy classes of loops in a reduced $G$-graph $\Gamma$. Represent each homotopy class $l_{i}$ by a reduced edge-path loop $u_{i}$ in $\Gamma$. We have defined the length $|L|_{\Gamma}$ to be the sum of the edge-path lengths of the $u_{i}$. It is convenient to assume that $L$ is $G$-equivariant (we can always arrange this by replacing $L$ by the finite set of its images under $G$ ).

A reduced edge-path in an arbitrary $G$-graph $\Gamma$ is still reduced after a forest collapse, so that the length of $L$ in $\Gamma$ decreases by the number of times edges of the forest are crossed by the path. The situation is more complicated under a fold, since a reduced edge-path may no longer be reduced after a fold. The neat way to keep track of all this is the following.

The star graph of $L$ with respect to $\Gamma$ has one vertex for each oriented edge of $\Gamma$, and one edge from $e$ to $f$ for each (cyclic) occurrence of $e \bar{f}$ in the edge path loops $u_{i}$. For a set of edges $E$ of $\Gamma$, we define $|E|$ to be the number of edges in the star graph for $L$ with one vertex in $E$ and one vertex not in $E$. For a Whitehead move $(\mathcal{I}, a)$, the total length of the edge paths representing $L$ in the new reduced marked graph changes by $\sum_{x \in G / P}|x \mathcal{I}|-|\{x a\}|$. Since $L$ is equivariant, this is just $p(|\mathcal{I}|-|\{a\}|)$, where $p$ is the index of $P$ in $G$. An equivariant Whitehead move $(\mathcal{I}, a)$ is reductive if $|\mathcal{I}|-|\{a\}| \leqslant 0$, and strictly reductive if $|\mathcal{I}|-|\{a\}|<0$.

\subsection{Counting}

To prove the equivariant peak reduction lemma, we use the counting technique developed in $[\mathbf{9}]$ and [10]. Below, $\Gamma$ is a reduced $G$-graph; in particular, $G$ acts on the set of oriented edges of $\Gamma$. 
Let $\mathcal{I}$ and $\mathcal{J}$ be two ideal edges in $\Gamma$, with stabilizers $P$ and $Q$, respectively. For $x \in G$, we have $x \mathcal{J} \cap \mathcal{J}$ is either empty or all of $\mathcal{J}$, and $x \mathcal{I} \cap \mathcal{I}$ is either empty or all of $\mathcal{I}$, since $\mathcal{I}$ and $\mathcal{J}$ are ideal edges. We consider the intersections $x \mathcal{J}$ with $\mathcal{I}$. If $x \mathcal{J} \cap \mathcal{I}=\emptyset$ for all $x \in G$, then $\mathcal{I}$ and $\mathcal{J}$ are compatible. If $\mathcal{I} \cap x \mathcal{J}$ is not empty, then $P(\mathcal{I} \cap x \mathcal{J})$ is called an intersection component of $\mathcal{I}$ with $G \mathcal{J}$. The correspondence $P(\mathcal{I} \cap x \mathcal{J}) \mapsto Q\left(\mathcal{J} \cap x^{-1} \mathcal{I}\right)$ can be used to show that the number of intersection components of $\mathcal{I}$ with $G \mathcal{J}$ is equal to the number of intersection components of $\mathcal{J}$ with $G \mathcal{I}$. This number is called the intersection number of $\mathcal{I}$ with $\mathcal{J}$.

Let $p=[G: P]$ and $q=[G: Q]$ be the indices of the stabilizers of $\mathcal{I}$ and $\mathcal{J}$ in $G$. The following lemmas are proved in $[\mathbf{1 0}]$.

Lemma 3.2. If the intersection number of $\mathcal{I}$ with $\mathcal{J}$ is 1 and $P \leqslant Q$, then

$$
p|\mathcal{I} \cap \mathcal{J}|+q|\mathcal{J} \cup Q \mathcal{I}| \leqslant p|\mathcal{I}|+q|\mathcal{J}| .
$$

Lemma 3.3. If $\gamma=\mathcal{I} \cap \mathcal{J}$ is not empty, then

$$
p|\mathcal{I}-P \gamma|+q|\mathcal{J}-Q \gamma| \leqslant p|\mathcal{I}|+q|\mathcal{J}| .
$$

\subsection{Equivariant splitting lemma}

Proposition 3.4. Let $\Gamma$ be a reduced $G$-graph. If $(\mathcal{I}, a)$ is a reductive and $(\mathcal{J}, b)$ a strictly reductive Whitehead move from $\Gamma$, then there is a sequence

$$
(\mathcal{I}, a)=\left(\mathcal{I}_{0}, a_{0}\right), \ldots,\left(\mathcal{I}_{k}, a_{k}\right)=(\mathcal{J}, b)
$$

of equivariant Whitehead moves such that $\left(\mathcal{I}_{i}, a_{i}\right)$ is strictly reductive for $i>0$, and $\mathcal{I}_{i}$ is compatible with $\mathcal{I}_{i+1}$ for $i=0, \ldots, k-1$.

Proof. The proof proceeds by induction on the intersection number $n$ of $\mathcal{I}$ with $\mathcal{J}$. If $n=0, \mathcal{I}$ and $\mathcal{J}$ are compatible, and there is nothing to prove. If $n>0$, our strategy will be to find a strictly reductive Whitehead move $(\mathcal{A}, c)$, so that the ideal edge $\mathcal{A}$ is compatible with $\mathcal{I}$ and has intersection number strictly less than $n$ with $\mathcal{J}$, or is compatible with $\mathcal{J}$ and has intersection number strictly less than $n$ with $\mathcal{I}$. The counting lemmas, 3.2 and 3.3 , will be used to prove strict reductivity.

Suppose there is an $x \in G$ with the following properties:

(1) $\mathcal{I} \cap x \mathcal{J} \neq \emptyset$;

(2) $a \notin \mathcal{I} \cap x \mathcal{J}$

(3) $x b \notin \mathcal{I} \cap x \mathcal{J}$.

Since $(x \mathcal{J}, x b)=(\mathcal{J}, b)$, without loss of generality we may assume $x=1$. Set $\gamma=\mathcal{I} \cap \mathcal{J}$. Since $a \notin \gamma$ and $P a=a$, we must have $a \notin P \gamma$; similarly, $b \notin Q \gamma$. Therefore, $(\mathcal{I}-P \gamma, a)$ and $(\mathcal{J}-Q \gamma, b)$ are Whitehead moves. The ideal edge $\mathcal{I}-P \gamma$ is compatible with $\mathcal{I}$ and has intersection number strictly smaller than $n$ with $\mathcal{J}$; similarly, $\mathcal{J}-Q \gamma$ is compatible with $\mathcal{J}$ and has intersection number strictly smaller than $n$ with $\mathcal{I}$. Lemma 3.3 gives

$$
p(|\mathcal{I}-P \gamma|-|a|)+q(|\mathcal{J}-Q \gamma|-|b|) \leqslant p(|\mathcal{I}|-|a|)+q(|\mathcal{J}|-|b|)<0,
$$




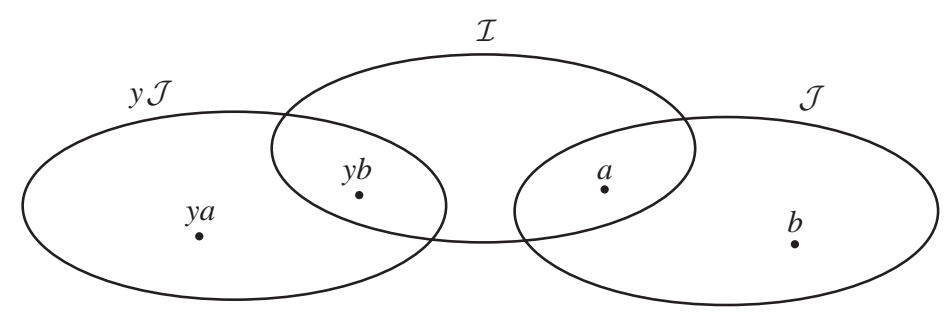

Figure 2. $n=2$.

showing at least one of these Whitehead moves is strictly reductive, so we are done by induction.

If there is no $x$ satisfying conditions (1)-(3), then every non-empty intersection $\mathcal{I} \cap x \mathcal{J}$ must contain either $a$ or $x b$. At most one intersection component $P(\mathcal{I} \cap x \mathcal{J})$ can contain $a$. Similarly, at most one intersection component $Q\left(\mathcal{J} \cap y^{-1} \mathcal{I}\right)$ can contain $b$, which translates to saying that at most one intersection component $P(\mathcal{I} \cap y \mathcal{J})$ meets the orbit of $b$. Therefore, there are at most two intersection components, i.e. $n \leqslant 2$.

If $n=2$, we must have $a \in \mathcal{I} \cap x \mathcal{J}$ and $y b \in \mathcal{I} \cap y \mathcal{J}$ for some $x, y$ in distinct cosets of $P$ in $G$. Without loss of generality, we may assume $x=1$, and $y \notin P$. Since $a \in \mathcal{J}$, we have $P=\operatorname{stab}(a) \leqslant Q=\operatorname{stab}(\mathcal{J})$. Since $y b \in \mathcal{I}$, we have $y Q y^{-1}=\operatorname{stab}(y b) \leqslant P$. But $P$ and $Q$ are finite groups, so $y Q y^{-1}=P=Q$. Set $\gamma=\mathcal{I} \cap \mathcal{J}$ and $\gamma_{y}=\mathcal{I} \cap y \mathcal{J}$. Since $a \in \gamma, \operatorname{stab}(a)=\operatorname{stab}(\gamma)$, i.e. $\gamma=P \gamma=Q \gamma$. Similarly, $\gamma_{y}=P \gamma_{y}=\left(y Q y^{-1}\right) \gamma_{y}$. The situation is illustrated in Figure 2.

We now divide the proof into cases depending on whether the orbits $G \bar{a}$ and $G \bar{b}$ meet $\mathcal{I} \cup \mathcal{J}$. Note that $G \bar{a}$ does not meet $\mathcal{I}$, by the definition of ideal edge, so that if $G \bar{a}$ meets $\mathcal{I} \cup \mathcal{J}$, it must meet it in $\mathcal{J}$.

If both $G \bar{a}$ and $G \bar{b}$ meet $\mathcal{I} \cup \mathcal{J}$, there are $x_{1}, x_{2} \in G$ with $x_{1} \bar{b} \in \mathcal{I}$ and $x_{2} \bar{a} \in \mathcal{J}$. Then $\left(\mathcal{I}-\gamma, x_{1} \bar{b}\right)$ and $\left(\mathcal{J}-\gamma, x_{2} \bar{a}\right)$ are Whitehead moves. The ideal edge $\mathcal{I}-\gamma$ is compatible with $\mathcal{I}$ and has intersection number 1 with $\mathcal{J}$; similarly, $\mathcal{J}-\gamma$ is compatible with $\mathcal{J}$ and has intersection number 1 with $\mathcal{I}$. An application of Lemma 3.3 shows that at least one of these is strictly reductive, so we are done by induction.

If only one of $G \bar{a}$ and $G \bar{b}$ meets $\mathcal{I} \cup \mathcal{J}$, by symmetry we may assume there is $x \in G$ with $x \bar{a} \in \mathcal{J}$, so that $(\mathcal{I}-\gamma, y b)$ and $(J-\gamma, x \bar{a})$ are Whitehead moves. We again apply Lemma 3.3 to show that at least one is strictly reductive.

If neither $G \bar{a}$ nor $G \bar{b}$ meets $\mathcal{I} \cup \mathcal{J}$, then $\left(y \mathcal{J}-\gamma_{y}, y a\right),\left(\mathcal{I}-\gamma_{y}, a\right),(\mathcal{I}-\gamma, y b)$ and $(\mathcal{J}-\gamma, b)$ are all Whitehead moves, and two applications of Lemma 3.3 give

$p\left(\left|y \mathcal{J}-\gamma_{y}\right|-|y a|+\left|\mathcal{I}-\gamma_{y}\right|-|a|+|\mathcal{I}-\gamma|-|y b|+|\mathcal{J}-\gamma|-|b|\right) \leqslant 2 p(|\mathcal{I}|-|a|+|\mathcal{J}|-|b|)<0$,

so that at least one of the Whitehead moves is strictly reductive.

We have now reduced to the case $n=1$. Without loss of generality, we may assume $\gamma=\mathcal{I} \cap \mathcal{J}$ is non-empty. Furthermore, at least one of $a$ or $b$ is in $\gamma$; otherwise conditions (1)-(3) are satisfied by $x=1$. By symmetry, we may assume $a \in \gamma$. Since $a \in \mathcal{J}$, $P=\operatorname{stab}(a) \leqslant Q=\operatorname{stab}(\mathcal{J})$. Thus, we have both counting lemmas, 3.3 and 3.2 , at our disposal. 

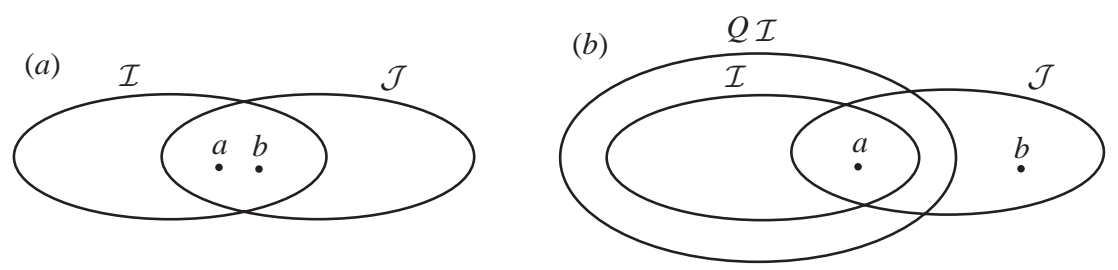

Figure 3. (a) $n=1, b \in \mathcal{I} \cap \mathcal{J}$. (b) $n=1, b \in \mathcal{J}-Q \mathcal{I}$.

There are two possibilities for the position of $b$. If $b \in \gamma$, then $b \in \mathcal{I}$, so $Q=\operatorname{stab}(b) \leqslant$ $P=\operatorname{stab}(\mathcal{I})$. But we already have $P \leqslant Q$, so $P=Q$ and $\gamma=P \gamma=Q \gamma$. If $b \notin \gamma$, then it is possible that $Q$ properly contains $P$ and $b \in \mathcal{J}-Q \gamma$. These possibilities are illustrated in Figure 3.

Again, we divide up the proof according to whether the orbits of $G \bar{a}$ or $G \bar{b}$ meet $\mathcal{I} \cup \mathcal{J}$. In each case, we will find a strictly reductive ideal edge compatible with both $\mathcal{I}$ and $\mathcal{J}$.

If both $G \bar{a}$ and $G \bar{b}$ meet $\mathcal{I} \cup \mathcal{J}$, there are $x_{1}, x_{2} \in G$ with $x_{1} \bar{b} \in \mathcal{I}$ and $x_{2} \bar{a} \in \mathcal{J}$. Since $G \bar{a} \cap \mathcal{I}=\emptyset$ and $G \bar{b} \cap \mathcal{J}=\emptyset, x_{1} \bar{b} \in \mathcal{I}-\gamma$ and $x_{2} \bar{a} \in \mathcal{J}-Q \gamma$. Then $\left(\mathcal{I}-\gamma, x_{1} \bar{b}\right)$ and $\left(\mathcal{J}-Q \gamma, x_{2} \bar{a}\right)$ are Whitehead moves. The ideal edges $\mathcal{I}-\gamma$ and $\mathcal{J}-Q \gamma$ are compatible with both $\mathcal{I}$ and $\mathcal{J}$, and an application of Lemma 3.3 finishes the proof in this case.

If $G \bar{b}$ does not meet $\mathcal{I} \cup \mathcal{J}$, then $(\gamma, a)$ and $(Q \mathcal{I} \cup \mathcal{J}, b)$ are Whitehead moves, and we can apply Lemma 3.2 to show that at least one is strictly reductive.

Finally, suppose $G \bar{b}$ meets $\mathcal{I} \cup \mathcal{J}$ but $G \bar{a}$ does not. If $b \in \gamma$, then $(\gamma, a)$ and $(\mathcal{I} \cup \mathcal{J}, b)$ are Whitehead moves, one of which is reductive by Lemma 3.2. If $b \in \mathcal{J}-Q \gamma$, choose $x \in G$ with $x \bar{b} \in \mathcal{I}-\gamma$. Then $\operatorname{stab}(x \bar{b})=\operatorname{stab}(x b)=x Q x^{-1} \leqslant \operatorname{stab}(\mathcal{I})=P$; but $P \leqslant Q$, so we must have $P=Q$ and $\gamma=P \gamma=Q \gamma$. Then $(\mathcal{I}-\gamma, x \bar{b}),(\mathcal{J}-\gamma, b),(\gamma, a)$ and $(\mathcal{I} \cup \mathcal{J}, a)$ are all Whitehead moves. We apply Lemma 3.3 to the first two and Lemma 3.2 to the last two to conclude

$$
|\mathcal{I}-\gamma|-|x \bar{b}|+|\mathcal{J}-\gamma|-|b|+|\gamma|-|a|+|\mathcal{I} \cup \mathcal{J}|-|a| \leqslant 2(|\mathcal{I}|-|a|+|\mathcal{J}|-|b|)<0,
$$

so that one of these Whitehead moves is strictly reductive.

\subsection{Equivariant peak reduction}

The following is a re-statement of Theorem 2.2 in the terminology of this section.

Theorem 3.5. Let $\Gamma$ be a reduced $G$-graph, and let $L$ be a finite invariant set of homotopy classes of loops in $\Gamma$. Let $\Gamma \rightarrow(\mathcal{I}, a) \Gamma$ and $\Gamma \rightarrow(\mathcal{J}, b) \Gamma$ be equivariant Whitehead moves with $(\mathcal{I}, a)$ reductive and $(\mathcal{J}, b)$ strictly reductive. Then there is an equivariant Whitehead path $\left(\mathcal{I}_{m}, a_{m}\right) \ldots\left(\mathcal{I}_{1}, a_{1}\right)$ from $(\mathcal{I}, a) \Gamma$ to $(\mathcal{J}, b) \Gamma$, such that the image of $L$ under $\left(\mathcal{I}_{j}, a_{j}\right) \ldots\left(\mathcal{I}_{1}, a_{1}\right)(\mathcal{I}, a)$ has length less than $|L|_{\Gamma}$ for all $1 \leqslant j \leqslant m$.

Proof. Suppose first that $\mathcal{I}$ and $\mathcal{J}$ are compatible. Then either their orbits are disjoint, the orbit $G \mathcal{I}$ is contained in $G \mathcal{J}$ (in which case we may assume $\mathcal{I} \subseteq \mathcal{J}$ ), or $G \mathcal{J}$ is contained in $G \mathcal{I}$ (in which case we may assume $\mathcal{J} \subseteq \mathcal{I}$ ). 
If $\mathcal{I}=\mathcal{J}$, then $(\mathcal{I}, a) \Gamma$ and $(\mathcal{J}, b) \Gamma$ are connected by a single Whitehead move, given by

$$
(\mathcal{I}, a) \Gamma \stackrel{c_{a}}{\longleftarrow} f_{\mathcal{I}}(\Gamma) \stackrel{c_{b}}{\longrightarrow}(\mathcal{I}, b) \Gamma .
$$

If $\mathcal{I}$ and $\mathcal{J}$ are different, we construct a new graph $\Gamma^{\prime}$ by performing both of the equivariant folds $f_{\mathcal{I}}$ and $f_{\mathcal{J}}$; if the orbits $G \mathcal{I}$ and $G \mathcal{J}$ are disjoint, these folds can be performed independently, in either order. If $\mathcal{I} \subset \mathcal{J}$ or $\mathcal{J} \subset \mathcal{I}$, we perform the fold corresponding to the larger orbit first. Now $\mathcal{I}, \mathcal{J}, a$ and $b$ correspond to edges in $\Gamma^{\prime}$, whose orbits are all forests. We set $\Phi_{a}=G\{a, \bar{a}\}$ and $\Phi_{b}=G\{b, \bar{b}\}$. If $\Phi_{a}=\Phi_{b}$, then

$$
(\mathcal{I}, a) \Gamma \stackrel{c_{J}}{\longleftarrow} c_{a}\left(\Gamma^{\prime}\right) \stackrel{c_{I}}{\longrightarrow}(\mathcal{J}, b) \Gamma
$$

is a single Whitehead move.

If $\Phi_{a}$ and $\Phi_{b}$ are different, and $\Phi=\Phi_{a} \cup \Phi_{b}$ is a forest in $\Gamma^{\prime}$, then the graph $\Gamma_{\Phi}^{\prime}$ obtained from $\Gamma^{\prime}$ by collapsing $\Phi$ is a reduced $G$-graph, and we have the following sequence of two Whitehead moves connecting $(\mathcal{I}, a) \Gamma$ and $(\mathcal{J}, b) \Gamma$ :

$$
(\mathcal{I}, a) \Gamma \stackrel{c_{J}}{\longleftarrow} c_{a}\left(\Gamma^{\prime}\right) \stackrel{c_{b}}{\longrightarrow} \Gamma_{\Phi}^{\prime} \stackrel{c_{a}}{\longleftarrow} c_{b}\left(\Gamma^{\prime}\right) \stackrel{c_{I}}{\longrightarrow}(\mathcal{J}, b) \Gamma .
$$

If $L_{\Phi}^{\prime}$ is the image of $L$ in $\Gamma_{\Phi}^{\prime}$, then

$$
\left|L_{\Phi}^{\prime}\right|=|L|_{\Gamma}+[G: \operatorname{stab}(\mathcal{I})](|\mathcal{I}|-|\{a\}|)+[G: \operatorname{stab}(\mathcal{J})](|\mathcal{J}|-|\{b\}|)<|L|_{\Gamma},
$$

and we are done.

We are reduced to the case when $\Phi=\Phi_{a} \cup \Phi_{b}$ is a not a forest in $\Gamma^{\prime}$.

Claim 3.6. If $\Phi$ is not a forest in $\Gamma^{\prime}$, then there are $x, y \in G$ so that either

(1) $(\mathcal{I}, x \bar{b})$ and $(\mathcal{J}, y \bar{a})$ are Whitehead moves; or

(2) $(\mathcal{I}, b)$ and $(\mathcal{J}, a)$ are Whitehead moves.

Proof. If $a$ has a free vertex in $\Phi$, then $\Phi$ deformation retracts onto $\Phi_{b}$, which is a forest in $\Gamma^{\prime}$, contradicting the hypothesis. Similarly, $b$ has no free vertex in $\Phi$.

Since $v_{\mathcal{I}}$ is not a free vertex of $\Phi_{a}$, there must be an edge of $\Phi$ different from $a$ which is in $\mathcal{I}$. We consider the different ways $\mathcal{I}$ could be compatible with $\mathcal{J}$ separately.

Case (1). Suppose $\mathcal{I} \cap x \mathcal{J}=\emptyset$ for all $x \in G$. Then there is no $x$ with $x b \in \mathcal{I}$. Since $\mathcal{I}$ is an ideal edge, there is no $x$ with $x \bar{a} \in \mathcal{I}$, or $x a \neq a$ in $\mathcal{I}$. Therefore, there must be an $x$ with $x \bar{b} \in \mathcal{I}$. Similarly, there must be a $y \in G$ with $y \bar{a} \in \mathcal{J}$. Therefore, both $(\mathcal{I}, x \bar{b})$ and $(\mathcal{J}, y \bar{a})$ are Whitehead moves.

Case (2). Suppose $\mathcal{I} \subset \mathcal{J}$. Then there is no $x$ with $x \bar{a}, x \bar{b}$ or $x a \neq a$ in $\mathcal{I}$.

Then we must have $b \in \mathcal{I}$. We know $x \bar{a} \notin \mathcal{I}$ for all $x$ but, in addition, $x \bar{a}$ cannot be in $\mathcal{J}-\mathcal{I}$, since that would imply that $x \bar{a}$ has $v_{\mathcal{J}}$ as a free vertex. Thus $(\mathcal{I}, b)$ and $(\mathcal{J}, a)$ are Whitehead moves.

The symmetric argument applies if $\mathcal{J} \subset \mathcal{I}$. 
In case $(1),(\mathcal{I}, x \bar{b})$ and $(\mathcal{J}, y \bar{a})$ are Whitehead moves, and $\operatorname{stab}(\mathcal{I})=\operatorname{stab}(\mathcal{J})$. Since $|\mathcal{I}|-|a|+|\mathcal{J}|-|b|<0$, one of $|\mathcal{I}|-|b|=|\mathcal{I}|-|x \bar{b}|$ or $|\mathcal{J}|-|y \bar{a}|$ is negative, so one of the Whitehead paths,

$$
(\mathcal{I}, a) \Gamma-(\mathcal{I}, x \bar{b}) \Gamma-(\mathcal{J}, b) \Gamma \quad \text { or } \quad(\mathcal{I}, a) \Gamma-(\mathcal{J}, y \bar{a}) \Gamma-(\mathcal{J}, b) \Gamma,
$$

satisfies the conclusion of the proposition.

Case (2) follows in the same way.

If $\mathcal{I}$ and $\mathcal{J}$ are not compatible, Proposition 3.4 (the splitting lemma) gives a sequence

$$
\mathcal{I}=\mathcal{I}_{0}, \mathcal{I}_{1}, \ldots, \mathcal{J}=\mathcal{I}_{k}
$$

of strictly reductive edges with $\mathcal{I}_{i}$ compatible with $\mathcal{I}_{i+1}$ for all $i$. Thus we can join each $\left(\mathcal{I}_{i}, a_{i}\right) \Gamma$ and $\left(\mathcal{I}_{i+1}, a_{i+1}\right) \Gamma$ by a Whitehead path that passes only through reduced $G$ graphs, such that the image of $L$ has length less than $|L|_{\Gamma}$.

The concatenation of all these paths gives a Whitehead path from $(\mathcal{I}, a) \Gamma$ to $(\mathcal{J}, b) \Gamma$ with the desired properties.

\section{Graphs of groups, their isomorphisms and Dehn twists}

In this section we review the basic terminology for graphs of groups and state some elementary facts. For more detailed information and proofs, we refer to $[\mathbf{4}]$ or $[\mathbf{1}]$.

\subsection{Graphs of groups}

A graph-of-groups $\mathcal{G}$ consists of the following data:

(1) a finite connected graph $\Gamma=\Gamma(\mathcal{G})$;

(2) for each vertex $v$ of $\Gamma$ a group $G_{v}$;

(3) for each oriented edge $e$ of $\Gamma$ a group $G_{e}$; if $\bar{e}$ denotes the edge corresponding to $e$ with the opposite orientation, then $G_{e}=G_{\bar{e}}$;

(4) for each oriented edge $e$ of $\Gamma$ an injective homomorphism $f_{e}: G_{e} \rightarrow G_{\tau(e)}$, where $\tau(e)$ is the terminal vertex of $e$.

In this paper, the following special class of graphs of groups will play a significant role.

Definition 4.1. A graph of groups $\mathcal{G}$ is free-cyclic if all vertex groups $G_{v}$ are free of rank at least two, all edge groups $G_{e}$ are infinite cyclic, and the image $f_{e}\left(G_{e}\right)$ of each edge group is a maximal cyclic subgroup of $G_{\tau(e)}$.

The path group $\Pi(\mathcal{G})$ is the group given by generators and relations as follows. As generators, we take the elements of the vertex groups $G_{v}$, together with one generator $t_{e}$ for each oriented edge $e$. As relations, we take all relations in the $G_{v}$ together with, for each oriented edge $e$ and element $a \in G_{e}$, the relations $t_{\bar{e}}=t_{e}^{-1}$ and $t_{e} f_{e}(a) t_{e}^{-1}=f_{\bar{e}}(a)$. 
Every element of $\Pi(\mathcal{G})$ is given by a word

$$
W=r_{0} t_{1} r_{1} \cdots t_{q} r_{q}
$$

where $t_{i}$ is equal to $t_{e_{i}}$ for some oriented edge $e_{i}$, and $r_{i}$ is an element of the free product of the vertex groups $G_{v}$. We say $W$ is a loop based at $v$ if the edges $e_{1} \ldots e_{q}$ form a loop in $\Gamma(\mathcal{G})$ at $v$ and the elements $r_{i}$ lie in the appropriate vertex groups, i.e. $\tau\left(\bar{e}_{1}\right)=\tau\left(e_{q}\right)=v$, $\tau\left(e_{i}\right)=\tau\left(\bar{e}_{i+1}\right)$ for $i=1, \ldots, q-1, r_{0} \in G_{v}$ and $r_{i} \in G_{\tau\left(e_{i}\right)}$ for $i=1, \ldots, q$. The fundamental group $\pi_{1}(\mathcal{G}, v)$ is defined to be the subset of $\Pi(\mathcal{G})$ consisting of elements that are represented by loops based at $v$. This is a subgroup of $\Pi(\mathcal{G})$. For distinct vertices $v_{1}$ and $v_{2}$, the subgroups $\pi_{1}\left(\mathcal{G}, v_{1}\right)$ and $\pi_{1}\left(\mathcal{G}, v_{2}\right)$ are conjugate in $\Pi(\mathcal{G})$.

\subsection{Graph-of-groups isomorphisms}

A graph-of-groups isomorphism $\mathcal{H}: \mathcal{G}_{1} \rightarrow \mathcal{G}_{2}$ consists of the following data:

(1) a graph isomorphism $H: \Gamma\left(\mathcal{G}_{1}\right) \rightarrow \Gamma\left(\mathcal{G}_{2}\right)$;

(2) for each vertex $v$ of $\Gamma\left(\mathcal{G}_{1}\right)$ an isomorphism $H_{v}: G_{v} \rightarrow G_{H(v)}$;

(3) for each oriented edge $e$ of $\Gamma\left(\mathcal{G}_{1}\right)$ an isomorphism $H_{e}: G_{e} \rightarrow G_{H(e)}$, with $H_{e}=H_{\bar{e}}$;

(4) for each edge $e$ of $\Gamma\left(\mathcal{G}_{1}\right)$ a correction term $\delta(e)=\delta_{H}(e)$; this is an element of $G_{H(v)}$ $(v=\tau(e))$ such that $H_{v} f_{e}=a d_{\delta(e)} f_{H(e)} H_{e}$, i.e. the following diagram commutes

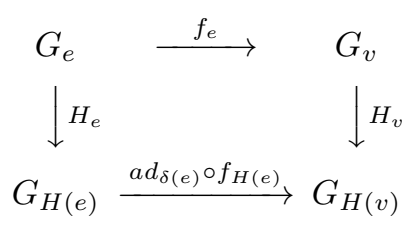

(Here and elsewhere, $a d_{x}$ denotes the conjugation $y \mapsto x y x^{-1}$.)

A graph-of-groups isomorphism $\mathcal{H}: \mathcal{G}_{1} \rightarrow \mathcal{G}_{2}$ induces an isomorphism $\mathcal{H}_{*}: \Pi\left(\mathcal{G}_{1}\right) \rightarrow$ $\Pi\left(\mathcal{G}_{2}\right)$ defined on generators by

$$
\begin{aligned}
\mathcal{H}_{*}(r) & =H_{v}(r), \quad \text { for } r \in G_{v} \\
\mathcal{H}_{*}\left(t_{e}\right) & =\delta(\bar{e}) t_{H(e)} \delta(e)^{-1}
\end{aligned}
$$

The isomorphism $\mathcal{H}_{*}$ in turn induces an isomorphism $\mathcal{H}_{* v}: \pi_{1}\left(\mathcal{G}_{1}, v\right) \rightarrow \pi_{1}\left(\mathcal{G}_{2}, H(v)\right)$.

Definition 4.2. Let $A$ and $B$ be groups. An outer isomorphism $F: A \rightarrow B$ is an equivalence class of isomorphisms, where two isomorphisms $f, f^{\prime}: A \rightarrow B$ are equivalent if there is an $x \in B$ with $f^{\prime}=a d_{x} \circ f$.

We denote by $\hat{\mathcal{H}}: \pi_{1}\left(\mathcal{G}_{1}\right) \rightarrow \pi_{1}\left(\mathcal{G}_{2}\right)$ the outer isomorphism induced by $\mathcal{H}_{* v}$. This notation that entirely omits the basepoints is justified by the observation that the isomorphisms $\mathcal{H}_{* v}$ and $\mathcal{H}_{* w}$ become equivalent upon identification of their source groups 
by a conjugacy in $\Pi\left(\mathcal{G}_{1}\right)$ and the identification of their target groups by a conjugacy in $\Pi\left(\mathcal{G}_{2}\right)($ see $[\mathbf{5}, \S 2])$.

The composition of two graph-of-groups isomorphisms $\mathcal{L}: \mathcal{G}_{1} \rightarrow \mathcal{G}_{2}$ and $\mathcal{K}: \mathcal{G}_{2} \rightarrow \mathcal{G}_{3}$ is a graph-of-groups isomorphism $\mathcal{H}: \mathcal{G}_{1} \rightarrow \mathcal{G}_{3}$, which satisfies $\hat{\mathcal{H}}=\hat{\mathcal{K}} \hat{\mathcal{L}}$ and $\mathcal{H}_{*}=\mathcal{K}_{*} \mathcal{L}_{*}$. The composition $\mathcal{H}$ is given by the compositions $H=K L, H_{v}=K_{L(v)} L_{v}, H_{e}=K_{L(e)} L_{e}$, and

$$
\delta_{H}(e)=K_{L(\tau(e))}\left(\delta_{L}(e)\right) \delta_{K}(L(e)),
$$

for all vertices $v$ and edges $e$ of $\Gamma_{1}$.

A graph-of-groups isomorphism between free-cyclic graphs of groups is determined to some extent by the graph isomorphism and the vertex isomorphisms. This is made precise in the following lemma.

Lemma 4.3. Let $\mathcal{G}_{1}$ and $\mathcal{G}_{2}$ be free-cyclic graphs of groups, and let $\mathcal{H}: \mathcal{G}_{1} \rightarrow \mathcal{G}_{2}$ and $\mathcal{H}^{\prime}: \mathcal{G}_{1} \rightarrow \mathcal{G}_{2}$ be two graph-of-groups isomorphisms which agree on the underlying graphs and on all vertex groups. Then $\mathcal{H}$ and $\mathcal{H}^{\prime}$ agree on the edge groups, and for each edge $e$ of $\mathcal{G}_{1}$, the correction terms $\delta(e)$ and $\delta^{\prime}(e)$ differ by the image of an element of the edge group $G_{H(e)}$ :

$$
\delta^{\prime}(e)=\delta(e) f_{H(e)}(b(e))
$$

for some $b(e) \in G_{H(e)}$.

Proof. Consider an edge $e$ of $\Gamma_{1}$, choose a generator $a$ for $G_{e}$ and let $v=\tau(e)$. Set $c=H_{e}(a)$ and $c^{\prime}=H_{e}^{\prime}(a)$; since $G_{H(e)}$ is infinite cyclic and $H_{e}$ and $H_{e}^{\prime}$ are isomorphisms, either $c^{\prime}=c$ or $c^{\prime}=c^{-1}$.

In the vertex group $G_{H(v)}, f_{H(e)}(c)$ is conjugate to $H_{v}\left(f_{e}(a)\right)$ by $\delta(e)$, and $f_{H^{\prime}(e)}\left(c^{\prime}\right)$ is conjugate to $H_{v}^{\prime}\left(f_{e}(a)\right)$ by $\delta^{\prime}(e)$. Since $H=H^{\prime}$ and $H_{v}=H_{v}^{\prime}, f_{H(e)}(c)$ is conjugate to $f_{H(e)}\left(c^{\prime}\right)$ by $\delta(e)^{-1} \delta^{\prime}(e)$. Since conjugation in the free group $G_{H(v)}$ cannot take an element to its inverse, we must have $c=c^{\prime}$, and, hence, $\delta(e)^{-1} \delta^{\prime}(e)$ commutes with $f_{H(e)}(c)$. Since the subgroup generated by $f_{H(e)}(c)$ is maximal cyclic in $G_{H(v)}$, the product $\delta(e)^{-1} \delta^{\prime}(e)$ is in this subgroup, and we take $b(e)$ so that $f_{H(e)}(b(e))=\delta(e)^{-1} \delta^{\prime}(e)$.

We will need to understand when two automorphisms of a graph of groups $\mathcal{G}$ induce conjugate elements of $\operatorname{Out}\left(\pi_{1}(\mathcal{G})\right)$. The following lemma describes one way that this may occur.

Lemma 4.4. Let $\mathcal{H}: \mathcal{G} \rightarrow \mathcal{G}$ be a graph-of-groups automorphism, let $e_{0}$ be an edge of $\Gamma(\mathcal{G})$ with $H\left(e_{0}\right) \neq e_{0}$, and let $b_{0}$ be an element of the centre of $G_{e_{0}}$. Let $\mathcal{H}^{\prime}: \mathcal{G} \rightarrow \mathcal{G}$ be the graph-of-groups automorphism obtained as follows. Set $e_{1}=H\left(e_{0}\right), e_{2}=H\left(e_{1}\right)$, $b_{1}=H_{e_{0}}\left(b_{0}\right)$ and $b_{2}=H_{e_{1}}\left(b_{1}\right)$; replace the correction term $\delta\left(e_{0}\right)$ by $\delta\left(e_{0}\right) f_{e_{1}}\left(b_{1}\right)$, and replace $\delta\left(e_{1}\right)$ by $\delta\left(e_{1}\right) f_{e_{2}}\left(b_{2}^{-1}\right)$. Leave all other data unchanged. Then $\hat{\mathcal{H}}$ and $\hat{\mathcal{H}}^{\prime}$ are conjugate in $\operatorname{Out}\left(\pi_{1}(\mathcal{G})\right)$.

Proof. Consider the graph-of-groups automorphism $\mathcal{D}: \mathcal{G} \rightarrow \mathcal{G}$ which is the identity on the graph and on all vertex and edge groups, and has trivial correction terms except for $\delta_{D}\left(e_{1}\right)=f_{e_{1}}\left(b_{1}\right)$. A straightforward computation shows that $D H=H^{\prime} D, D_{v} H_{v}=H_{v}^{\prime} D_{v}$ 
and $D_{e} H_{e}=H_{e}^{\prime} D_{e}$ for all vertices $v$ and edges $e$. We perform here the calculations for the correction terms $\delta(e)$, for an arbitrary edge $e$ of $\Gamma(\mathcal{G})$ with terminal vertex $\tau(e)=v$ :

$$
\begin{gathered}
\delta_{D H}(e)=D_{H(v)}\left(\delta_{H}(e)\right) \delta_{D}(H(e))=\delta_{H}(e) \delta_{D}(H(e)), \\
\delta_{H^{\prime} D}(e)=H_{D(v)}^{\prime}\left(\delta_{D}(e)\right) \delta_{H^{\prime}}(D(e))=H_{v}^{\prime}\left(\delta_{D}(e)\right) \delta_{H^{\prime}}(e) .
\end{gathered}
$$

These are both equal to $\delta_{H}(e)$ if $e \neq e_{0}, e_{1}$. For $e=e_{0}$ one obtains

$$
\begin{aligned}
\delta_{D H}\left(e_{0}\right) & =\delta_{H}\left(e_{0}\right) \delta_{D}\left(e_{1}\right)=\delta_{H}\left(e_{0}\right) f_{e_{1}}\left(b_{1}\right), \\
\delta_{H^{\prime} D}\left(e_{0}\right) & =H_{\tau\left(e_{0}\right)}^{\prime}\left(\delta_{D}\left(e_{0}\right)\right) \delta_{H^{\prime}}\left(e_{0}\right)=\delta_{H}\left(e_{0}\right) f_{e_{1}}\left(b_{1}\right),
\end{aligned}
$$

and for $e=e_{1}$, one has

$$
\begin{aligned}
\delta_{D H}\left(e_{1}\right) & =\delta_{H}\left(e_{1}\right) \delta_{D}\left(e_{2}\right)=\delta_{H}\left(e_{1}\right), \\
\delta_{H^{\prime} D}\left(e_{1}\right) & =H_{\tau\left(e_{1}\right)}^{\prime}\left(\delta_{D}\left(e_{1}\right)\right) \delta_{H^{\prime}}\left(e_{1}\right)=H_{\tau\left(e_{1}\right)}^{\prime}\left(f_{e_{1}}\left(b_{1}\right)\right) \delta_{H^{\prime}}\left(e_{1}\right) \\
& =\left[\delta_{H^{\prime}}\left(e_{1}\right) f_{e_{2}}\left(b_{2}\right) \delta_{H^{\prime}}\left(e_{1}\right)^{-1}\right] \delta_{H^{\prime}}\left(e_{1}\right)=\delta_{H^{\prime}}\left(e_{1}\right) f_{e_{2}}\left(b_{2}\right) \\
& =\delta_{H}\left(e_{1}\right) f_{e_{2}}\left(b_{2}{ }^{-1}\right) f_{e_{2}}\left(b_{2}\right)=\delta_{H}\left(e_{1}\right) .
\end{aligned}
$$

Thus, $\mathcal{D H}=\mathcal{H}^{\prime} \mathcal{D}$, and so the outer automorphisms induced by $\mathcal{H}$ and $\mathcal{H}^{\prime}$ are conjugate.

The graph-of-groups isomorphism $\mathcal{D}$ in the proof of the above lemma induces a 'Dehn twist automorphism' $\hat{\mathcal{D}} \in \operatorname{Out}\left(\pi_{1}(\mathcal{G})\right)$. We now give a precise definition of this.

Definition 4.5. A Dehn twist $D$ consists of the following data:

(1) a graph of groups $\mathcal{G}$;

(2) for each edge $e$ of $\Gamma=\Gamma(\mathcal{G})$, an element $z_{e}$ in the centre of the edge group $G_{e}$, with $z_{\bar{e}}=z_{e}^{-1}$.

We say $D$ is based on $\mathcal{G}$, and the elements $z_{e}$ are called twistors.

The Dehn twist $D$ determines an automorphism $D_{*}: \Pi(\mathcal{G}) \rightarrow \Pi(\mathcal{G})$ given on the generators by $\left.D_{*}\right|_{G_{v}}=$ id for each vertex $v \in \Gamma$, and $D_{*}\left(t_{e}\right)=t_{e} f_{e}\left(z_{e}\right)$ for each edge $e \in \Gamma$. The automorphism $D_{*}$ restricts to an automorphism $D_{v}: \pi_{1}(\mathcal{G}, v) \rightarrow \pi_{1}(\mathcal{G}, v)$ for every vertex $v$ of $\Gamma$ and, hence, defines an outer automorphism $\hat{D}$ of $\pi_{1}(\mathcal{G})$, which we call a Dehn twist automorphism.

A graph-of-groups automorphism $\mathcal{D}$ represents a Dehn twist $D$ based on $\mathcal{G}$ if $\mathcal{D}$ is an automorphism of $\mathcal{G}$ with $\hat{\mathcal{D}}=\hat{D}$ in $\operatorname{Out}\left(\pi_{1}(\mathcal{G})\right)$. We can easily construct a representative of $D$ by taking the identity on the graph and on all vertex and edge groups, and taking correction terms any elements $\delta(e) \in G_{\tau(e)}$ satisfying $\delta(\bar{e}) t_{e} \delta(e)^{-1}=t_{e} f_{e}\left(z_{e}\right)$ in $\Pi(\mathcal{G})$, where $\left\{z_{e}\right\}$ are the twistors of $D$. The following proposition characterizes all representatives of a Dehn twist, when the graph of groups $\mathcal{G}$ is free-cyclic. 


\section{Proposition 4.6.}

(1) Let $\mathcal{G}$ be a free-cyclic graph of groups, let $D$ be a Dehn twist based on $\mathcal{G}$, and let $\mathcal{D}: \mathcal{G} \rightarrow \mathcal{G}$ represent $D$. Then $\mathcal{D}$ is the identity on the underlying graph and on all edge groups, and for all vertices $v$ and edges e, there exist elements $\gamma(v) \in G_{v}$ and $b(e) \in G_{e}$ such that $D_{v}=a d_{\gamma(v)}$ and $\delta(e)=\gamma(\tau(e)) f_{e}(b(e))$.

(2) Let $\mathcal{G}$ be any graph of groups, with given elements $\gamma(v)$ in each vertex group $G_{v}$ and $b(e)$ in each edge group $G_{e}$. Let $\mathcal{D}: \mathcal{G} \rightarrow \mathcal{G}$ be the graph-of-groups automorphism that is the identity on $\Gamma(\mathcal{G})$ and on all edge groups, is $a d_{\gamma(v)}$ on $G_{v}$, and has correction terms $\delta(e)=\gamma(\tau(e)) f_{e}(b(e))$. Then $\mathcal{D}$ represents the Dehn twist $D^{\prime}$ based on $\mathcal{G}$ with twistors $\left\{z_{e}=b(\bar{e}) b(e)^{-1}\right\}$.

Proof. (1) Since every vertex group $G_{v}$ is free of rank at least two, and since $\Gamma(\mathcal{G})$ is a finite graph, there is an element $r(v) \in G_{v}$ which is not conjugate to any element of any $f_{e}\left(G_{e}\right)$. If follows from the normal form of cyclic words in $\Pi(\mathcal{G})$ (see [5, Proposition 3.9]) that the conjugacy class $\hat{\mathcal{D}}[r(v)]=\hat{D}[r(v)]$ in $\Pi(\mathcal{G})$ is equal to $[r(v)]$. This implies that $D(v)=v$. A similar consideration for the conjugacy class $\left[t_{e} r(\tau(e)) t_{\bar{e}} r(\tau(\bar{e}))\right]$ implies that $D(e)=e$, and, thus, $\mathcal{D}$ is the identity on $\Gamma(\mathcal{G})$. But then every $D_{v}$ is an inner automorphism of $G_{v}$, say $D_{v}=a d_{\gamma(v)}$ for $\gamma(v) \in G_{v}$, and every $D_{e}$ is the identity map. As every edge group $G_{e}$ is mapped by $f_{e}$ to a maximal cyclic subgroup of the non-abelian free group $G_{\tau(e)}$, it follows from

$$
a d_{\gamma(\tau(e))} f_{e}=D_{\tau(e)} f_{e}=a d_{\delta(e)} f_{D(e)} D_{e}=a d_{\delta(e)} f_{e}
$$

that $\delta(e)$ differs from $\gamma(\tau(e))$ at most by an element from $f_{e}\left(G_{e}\right)$, i.e. $\delta(e)=\gamma(\tau(e)) f_{e}(b(e))$ for some $b(e) \in G_{e}$.

(2) This follows directly if we compare the definition of $\mathcal{D}_{*}(W)$ and $D_{*}^{\prime}(W)$ for an arbitrary word $W$ in the path group $\Pi(\mathcal{G})$.

Corollary 4.7. Let $\mathcal{G}$ be a free-cyclic graph of groups, let $D$ be a Dehn twist based on $\mathcal{G}$, let $\mathcal{D}: \mathcal{G} \rightarrow \mathcal{G}$ represent $D$, and let $b(e) \in G_{e}$ be the elements found in Proposition 4.6(1). Then the twistors of $D$ are determined by the correction terms of $\mathcal{D}$ via the formula

$$
z_{e}=b(\bar{e}) b(e)^{-1} \text {. }
$$

Proof. By Proposition 4.6(2), $\mathcal{D}$ represents the Dehn twist $D^{\prime}$ based on $\mathcal{G}$ with twistors $z_{e}=b(\bar{e}) b(e)^{-1}$. By [5, Proposition 5.4], a Dehn twist on a free-cyclic graph of groups is uniquely determined by the induced outer automorphism of $\pi_{1}(\mathcal{G})$. Since $\hat{D}=\hat{\mathcal{D}}=\hat{D}^{\prime}$, we must have $D=D^{\prime}$.

Applying Proposition 4.6 in the special case when the Dehn twist is trivial results in the following two useful corollaries.

Corollary 4.8. Let $\mathcal{H}: \mathcal{G} \rightarrow \mathcal{G}$ be a graph-of-groups automorphism, $w$ a vertex of $\Gamma(\mathcal{G})$, and $\gamma$ an element of the vertex group $G_{H(w)}$. Let $\mathcal{H}_{\gamma}: \mathcal{G} \rightarrow \mathcal{G}$ be the graphof-groups automorphism obtained from $\mathcal{H}$ as follows. Replace $H_{w}: G_{w} \rightarrow G_{H(w)}$ by 
$a d_{\gamma} \circ H_{w}$, and for each edge e terminating at $w$, replace $\delta(e)$ by $\gamma \delta(e)$. Then $\mathcal{H}$ and $\mathcal{H}_{\gamma}$ induce the same outer automorphism of $\pi_{1}(\mathcal{G})$.

Proof. Let $\mathcal{D}$ be the graph-of-groups automorphism defined as in Proposition 4.6(2) by setting $b(e)=1$ for all edges $e, \gamma(v)=\gamma$ if $v=H(w)$, and $\gamma(v)=1$ otherwise. Then $\mathcal{H}_{\gamma}=\mathcal{D} H$. But $\hat{\mathcal{D}}$ is trivial in $\operatorname{Out}\left(\pi_{1}(\mathcal{G})\right)$ by Proposition $4.6(2)$.

Corollary 4.9. Let $\mathcal{G}$ be a free-cyclic graph of groups. If two graph-of-groups automorphisms $\mathcal{H}: \mathcal{G} \rightarrow \mathcal{G}$ and $\mathcal{K}: \mathcal{G} \rightarrow \mathcal{G}$ induce the same outer automorphism of $\pi_{1}(\mathcal{G})$, then

(1) $H=K: \Gamma(\mathcal{G}) \rightarrow \Gamma(\mathcal{G})$; and

(2) for each vertex $v$ of $\Gamma(\mathcal{G}), H_{v}$ and $K_{v}$ induce the same outer isomorphism $G_{v} \rightarrow$ $G_{H(v)}$.

Proof. Apply part (1) of Proposition 4.6 to $\mathcal{D}=\mathcal{K H}^{-1}$; we know that $\hat{\mathcal{D}}$ is the identity.

For the rest of this paper, we specialize to graphs of groups $\mathcal{G}$ with finitely generated free fundamental group $F_{n}$. We say a graph-of-groups automorphism $\mathcal{H}: \mathcal{G} \rightarrow \mathcal{G}$ realizes an element $\phi$ of $\operatorname{Out}\left(F_{n}\right)$ if there is an outer isomorphism $\theta: F_{n} \rightarrow \pi_{1}(\mathcal{G})$ with $\theta^{-1} \hat{\mathcal{H}} \theta=\phi$. An element $\phi \in \operatorname{Out}\left(F_{n}\right)$ is called a Dehn twist automorphism if there is a graph-ofgroups automorphism realizing $\phi$ that represents a Dehn twist $D$; in this case we also say $D$ realizes $\phi$.

In general, a given Dehn twist automorphism of $F_{n}$ may be realized by many different Dehn twists, based on different graphs of groups. A Dehn twist based on $\mathcal{G}$ is called efficient if $\mathcal{G}$ is free-cyclic with free fundamental group, the twistors $z_{e}$ are all nontrivial, and the images of two twistors in the same vertex group have no positive powers that are conjugate in the vertex group (see $[\mathbf{5}, \S 6]$ ). In $[\mathbf{5}]$ it is shown that every Dehn twist automorphism of $F_{n}$ is realized by an efficient Dehn twist. The main result of [5] (Theorem 1.1(a)) is the following uniqueness result for efficient Dehn twists.

Theorem 4.10. Let $\mathcal{G}_{1}$ and $\mathcal{G}_{2}$ be graphs of groups with $\pi_{1}\left(\mathcal{G}_{1}\right) \cong \pi_{1}\left(\mathcal{G}_{2}\right) \cong F_{n}$. Let $D_{1}$ and $D_{2}$ be efficient Dehn twists based on $\mathcal{G}_{1}$ and $\mathcal{G}_{2}$, respectively, with twistors $\left\{z_{e}\right\}$ and $\left\{z_{e}^{\prime}\right\}$, and let $\eta: \pi_{1}\left(\mathcal{G}_{1}\right) \rightarrow \pi_{1}\left(\mathcal{G}_{2}\right)$ be an outer isomorphism. Then the outer automorphisms $\hat{D}_{2}$ and $\eta \hat{D}_{1} \eta^{-1}$ of $\pi_{1}\left(\mathcal{G}_{2}\right)$ are equal if and only if there is a graphof-groups isomorphism $\mathcal{H}: \mathcal{G}_{1} \rightarrow \mathcal{G}_{2}$ with $\hat{\mathcal{H}}=\eta$ that takes twistors to twistors (i.e. $H_{e}\left(z_{e}\right)=z_{H(e)}^{\prime}$ for all edges $\left.e \in \Gamma\left(\mathcal{G}_{1}\right)\right)$.

\section{Roots of Dehn twists}

In this section we consider graphs of groups $\mathcal{G}$ with free fundamental group and outer automorphisms $\phi \in \operatorname{Out}\left(\pi_{1}(\mathcal{G})\right)$ such that some positive power $\phi^{t}$ is a Dehn twist automorphism. We give a solution to the conjugacy problem for such roots of Dehn twist automorphisms, based on the equivariant Whitehead algorithm derived in $\S 2$. 


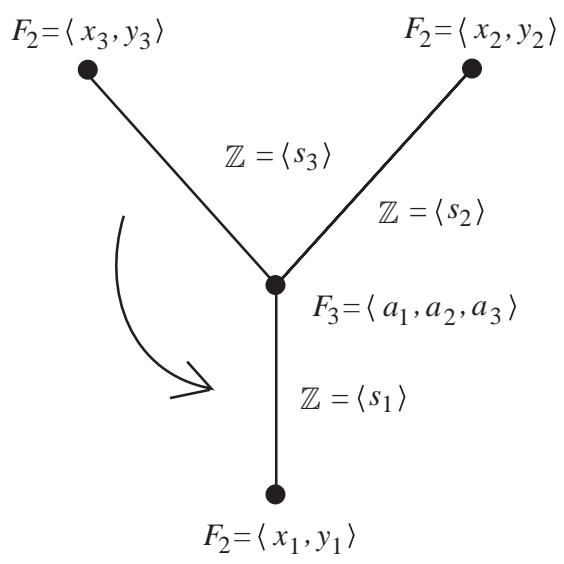

Figure 4. Example 5.1.

To help the reader understand the main problems in dealing with roots of Dehn twist automorphisms, we revisit the example given in the introduction to this paper. We describe two graph-of-groups automorphisms that realize the same automorphisms of the free group as those induced by the surface homeomorphisms in that example.

Example 5.1. Let $\Gamma$ be the 'tripod' graph, with four vertices $\left\{v_{1}, v_{2}, v_{3}, w\right\}$ and three edges $\left\{e_{1}, e_{2}, e_{3}\right\}$, with $e_{i}$ connecting $v_{i}$ to $w$. Let $\mathcal{G}$ be the graph of groups based on $\Gamma$, with $G_{w}=\left\langle a_{1}, a_{2}, a_{3}\right\rangle \cong F_{3}, G_{v_{i}}=\left\langle x_{i}, y_{i}\right\rangle \cong F_{2}, G_{e_{i}}=\left\langle s_{i}\right\rangle \cong \mathbb{Z}, f_{e_{i}}\left(s_{i}\right)=a_{i}$, and $f_{\bar{e}_{i}}\left(s_{i}\right)=\left[x_{i}, y_{i}\right]$ (see Figure 4$)$.

Define a graph-of-groups isomorphism $\mathcal{R}$ as follows, where all indices should be interpreted modulo 3 . The graph isomorphism $R$ cyclically permutes the edges, sending $e_{i} \mapsto e_{i+1}$ and fixing the central vertex $w$; the vertex group isomorphism $R_{w}$ sends each $a_{i}$ to $a_{i+1}$, and the vertex group isomorphisms $R_{v_{i}}: G_{v_{i}} \rightarrow G_{v_{i+1}}$ are given by $x_{i} \mapsto x_{i+1}$ and $y_{i} \mapsto y_{i+1}$; the edge isomorphisms $R_{e_{i}}$ are given by $s_{i} \mapsto s_{i+1}$; the correction terms are given by $\delta\left(\bar{e}_{i}\right)=1$ and $\delta\left(e_{i}\right)=a_{i+1}^{-1}$. Define a second graph-of-groups isomorphism $\mathcal{R}^{\prime}$ with exactly the same graph, vertex group and edge group isomorphisms, but with all correction terms trivial except $\delta\left(e_{1}\right)=a_{2}^{-3}$.

Then $\mathcal{R}^{3}=\mathcal{R}^{\prime 3}$, and this represents an (efficient!) Dehn twist with twistors $\left\{z_{e_{i}}=s_{i}^{3}\right\}$. Are the automorphisms $\hat{\mathcal{R}}$ and $\hat{\mathcal{R}}^{\prime}$ of $\pi_{1}(\mathcal{G})$ conjugate? The answer is yes; in fact, $\mathcal{R}$ and $\mathcal{R}^{\prime}$ are conjugate by a Dehn twist $D$ which twists once around $s_{2}\left(\right.$ i.e. $\left.\delta\left(e_{2}\right)=a_{2}^{-1}\right)$ and twice around $s_{3}\left(\delta\left(e_{3}\right)=a_{3}^{-2}\right)$, but not around $s_{1}\left(\delta\left(e_{1}\right)=1\right)$.

We begin by deriving, from results of [5], the fact that a root of a Dehn twist automorphism is realized by a particularly simple automorphism of a graph of groups, related to the efficient representative for the associated Dehn twist automorphism.

Definition 5.2. Let $D$ be a Dehn twist based on the graph of groups $\mathcal{G}$, with twistors $\left\{z_{e}\right\}$. A graph-of-groups automorphism $\mathcal{R}: \mathcal{G} \rightarrow \mathcal{G}$ is a $k$-th root of $D$ if $\mathcal{R}^{k}$ represents $D$ and $R_{e}\left(z_{e}\right)=z_{R(e)}$ for every edge $e$ of $\mathcal{G}$. 
Proposition 5.3. Let $\phi$ be an element of $\operatorname{Out}\left(F_{n}\right)$ such that $\phi^{t}$ is a Dehn twist automorphism. Then $\phi$ is realized by a root of an efficient Dehn twist.

Proof. By Corollary 8.7 of [5], there is an efficient Dehn twist $D$ based on $\mathcal{G}$ with twistors $\left\{z_{e}\right\}$ which realizes $\phi^{t}$. Since $\phi$ commutes with $\phi^{t}$, Proposition 7.1 of [5] implies that there is a graph-of-groups automorphism $\mathcal{R}: \mathcal{G} \rightarrow \mathcal{G}$ realizing $\phi$ with $R_{e}\left(z_{e}\right)=z_{R(e)}$ for all edges $e$ of $\Gamma(\mathcal{G})$.

Remark 5.4. Proposition 5.3 can be used to derive basic information about roots of Dehn twists, for example about their centralizer in $\operatorname{Out}\left(F_{n}\right)$ or about the fixed subgroups of their preferred lifts to Aut $\left(F_{n}\right)$, from the analogous statements for Dehn twists (see [5, $\S 7])$.

The following technical lemma shows the effect of a simple alteration of a single correction term in a graph-of-groups automorphism.

Lemma 5.5. Let $\mathcal{R}: \mathcal{G} \rightarrow \mathcal{G}$ be a graph-of-groups automorphism. Let $e_{0}$ be an edge of $\Gamma(\mathcal{G})$ and $c_{0}$ an element of $G_{e_{0}}$. Set $e_{k}=R^{k}\left(e_{0}\right)$ and $c_{k}=\left(R^{k}\right)_{e_{0}}\left(c_{0}\right)$, and let $E=\left\{e_{0}, e_{1}, \ldots, e_{t\left(e_{0}\right)-1}\right\}$ be the orbit of $e_{0}$ and $s=t / t\left(e_{0}\right)$. Define $\mathcal{R}_{1}: \mathcal{G} \rightarrow \mathcal{G}$ to be the graph-of-groups automorphism obtained from $\mathcal{R}$ by replacing the correction term $\delta\left(e_{0}\right)$ with $\delta\left(e_{0}\right) f_{e_{1}}\left(c_{1}\right)$. Then

$$
\delta_{\mathcal{R}_{1}^{t}}\left(e_{j}\right)=\delta_{\mathcal{R}^{t}}\left(e_{j}\right) f_{e_{j}}\left(c_{j}\right)^{s}
$$

and

$$
\delta_{\mathcal{R}_{1}^{t}}(e)=\delta_{\mathcal{R}^{t}}(e), \quad \text { if } e \notin E .
$$

If $\mathcal{G}$ is free-cyclic and $\mathcal{R}^{t}$ represents a Dehn twist, then $\mathcal{R}_{1}^{t}$ also represents a Dehn twist.

Proof. We compute the correction terms for $\mathcal{R}^{t}$ from those of $\mathcal{R}$ using the formula

$$
\delta_{H}(e)=K_{L(\tau(e))}\left(\delta_{L}(e)\right) \delta_{K}(L(e))
$$

for a composition $\mathcal{H}=\mathcal{K} \mathcal{L}$ of graph-of-groups isomorphisms. We omit subscripts that are well defined by the context:

$$
\begin{aligned}
\delta_{R^{t}}(e) & =R^{t-1}(\delta(e)) \delta_{R^{t-1}}(R(e)) \\
& =R^{t-1}(\delta(e)) R^{t-2}(\delta(R(e))) \delta_{R^{t-2}}\left(R^{2}(e)\right) \\
& =\cdots \\
& =R^{t-1}(\delta(e)) R^{t-2}(\delta(R(e))) \cdots R^{t-k-1}\left(\delta\left(R^{k}(e)\right)\right) \cdots \delta\left(R^{t-1}(e)\right) .
\end{aligned}
$$

In order to obtain the correction terms for $\mathcal{R}_{1}^{t}$, each occurrence of $\delta\left(e_{0}\right)$ in the above expression must be replaced by $\delta\left(e_{0}\right) f_{e_{1}}\left(c_{1}\right)$. If $e \notin E$, then $\delta\left(e_{0}\right)$ does not occur, and we have $\delta_{R_{1}^{t}}(e)=\delta_{R^{t}}(e)$. 
If $e \in E$, then $\delta\left(e_{0}\right)$ occurs $s=t / t\left(e_{0}\right)$ times. For each $k$ with $R^{k}(e)=e_{0}$ we apply the rule $R f_{e}=a d_{\delta(e)} f R_{e}$ from the definition of a graph-of-groups isomorphism repeatedly to obtain

$$
\begin{aligned}
R^{t-k-1}\left(\delta\left(e_{0}\right) f_{e_{1}}\left(c_{1}\right)\right)= & R^{t-k-1}\left(\delta\left(e_{0}\right)\right) R^{t-k-1}\left(f_{e_{1}}\left(c_{1}\right)\right) \\
& =R^{t-k-1}\left(\delta\left(e_{0}\right)\right) R^{t-k-2}\left(R f_{e_{1}}\left(c_{1}\right)\right) \\
& =R^{t-k-1}\left(\delta\left(e_{0}\right)\right) R^{t-k-2}\left(\delta\left(e_{1}\right) f_{e_{2}}\left(c_{2}\right) \delta\left(e_{1}\right)^{-1}\right) \\
& =R^{t-k-1}\left(\delta\left(e_{0}\right)\right) R^{t-k-2}\left(\delta\left(e_{1}\right)\right) R^{t-k-2}\left(f_{e_{2}}\left(c_{2}\right)\right) R^{t-k-2}\left(\delta\left(e_{1}\right)\right)^{-1} \\
& =\cdots \quad \\
& =R^{t-k-1}\left(\delta\left(e_{0}\right)\right) R^{t-k-2}\left(\delta\left(e_{1}\right)\right) R^{t-k-3}\left(\delta\left(e_{2}\right)\right) \cdots \\
& \quad \cdots \delta\left(e_{t-k-1}\right) f_{e_{t-k}}\left(c_{t-k}\right) \delta\left(e_{t-k-1}\right)^{-1} \cdots \\
& \cdots R^{t-k-3}\left(\delta\left(e_{2}\right)\right)^{-1} R^{t-k-2}\left(\delta\left(e_{1}\right)\right)^{-1}
\end{aligned}
$$

Since $R^{k}(e)=e_{0}$, we have $e_{t-k}=e_{t\left(e_{0}\right)-k}=e$ (and, hence, $c_{t-k}=c_{t\left(e_{0}\right)-k}$ ). For $j=t\left(e_{0}\right)-k$, it follows that $\delta_{R_{1}^{t}}\left(e_{j}\right)=\delta_{R^{t}}\left(e_{j}\right) f_{e_{j}}\left(c_{j}\right)^{s}$.

If $\mathcal{G}$ is free-cyclic and $\mathcal{R}^{t}$ represents a Dehn twist, then the correction terms of $\mathcal{R}^{t}$ are determined by elements $\gamma(v)$ and $b(e)$ as in Proposition 4.6(1). The above computation of the correction terms for $\mathcal{R}_{1}^{t}$ together with Proposition 4.6(2) then imply that $\mathcal{R}_{1}^{t}$ also represents a Dehn twist.

We use the above lemma as a tool in the next proposition, which says that two roots of an efficient Dehn twist that agree on most of their data must induce conjugate outer automorphisms.

Proposition 5.6. Let $\mathcal{G}$ be free-cyclic, and let $\mathcal{R}$ and $\mathcal{R}^{\prime}$ be $t$-th roots of an efficient Dehn twist based on $\mathcal{G}$ with twistors $\left\{z_{e}\right\}$. If $\mathcal{R}$ and $\mathcal{R}^{\prime}$ agree on the graph and on all vertex groups of $\mathcal{G}$, then $\hat{\mathcal{R}}$ and $\hat{\mathcal{R}}^{\prime}$ are conjugate in $\operatorname{Out}\left(\pi_{1}(\mathcal{G})\right)$.

Proof. We will modify $\mathcal{R}$ without changing the conjugacy class of $\hat{\mathcal{R}}$ until $\mathcal{R}=\mathcal{R}^{\prime}$.

For any edge $e$ of $\Gamma(\mathcal{G})$, Lemma 4.3 guarantees that $\mathcal{R}$ and $\mathcal{R}^{\prime}$ agree on the edge groups, and gives us an element $b(e) \in G_{e}$ such that the correction terms $\delta(e)$ of $\mathcal{R}$ and $\delta^{\prime}(e)$ of $\mathcal{R}^{\prime}$ are related by

$$
\delta^{\prime}(e)=\delta(e) f_{R(e)} R_{e}(b(e)) .
$$

Lemma 4.4 then implies that, if $R(e) \neq e$, we may replace $\delta(e)$ in $\mathcal{R}$ by $\delta^{\prime}(e)$ and $\delta(R(e))$ by

$$
\delta(R(e)) f_{R(R(e))}\left(R_{R(e)} R_{e}\left(b(e)^{-1}\right)\right),
$$

without changing the conjugacy class of $\hat{\mathcal{R}}$.

Choose an edge $e$ in each non-trivial edge orbit, and perform the double replacement above. We may then replace the new $\delta(R(e))$ by $\delta^{\prime}(R(e))$ at the cost of modifying $\delta(R(R(e)))$. We continue through each edge orbit, modifying the correction terms for $\mathcal{R}$ until they agree with those of $\mathcal{R}^{\prime}$ except, possibly, at one exceptional edge of each orbit. 
Let $e$ be an exceptional edge, with $\delta^{\prime}(e)=\delta(e) f_{R(e)}\left(R_{e}(b(e))\right)$ as above. Suppose first that $\bar{e}$ is in the orbit of $e$, say $\bar{e}=R^{k}(e)$. By Lemma 5.5, we have

$$
\begin{aligned}
& \delta_{\mathcal{R}^{\prime t}}(e)=\delta_{\mathcal{R}^{t}}(e) f_{e}\left(b(e)^{s}\right), \\
& \delta_{\mathcal{R}^{\prime t}}(\bar{e})=\delta_{\mathcal{R}^{t}}(\bar{e}) f_{\bar{e}}\left(R_{e}^{k}(b(e))^{s}\right) .
\end{aligned}
$$

Since $\mathcal{R}$ is a root of an efficient Dehn twist, the twistor $z_{e}$ is non-trivial; since $R_{e}\left(z_{e}\right)=$ $z_{R(e)}$, we have $R_{e}^{k}\left(z_{e}\right)=z_{R^{k}(e)}=z_{\bar{e}}=z_{e}^{-1}$, so that $R_{e}^{k}$ acts as inversion on the edge group $G_{e}$. In particular, $R_{e}^{k}(b(e))^{s}=b(e)^{-s}$.

Since $\mathcal{R}^{t}$ and $\mathcal{R}^{\prime t}$ represent the same efficient Dehn twist and agree on all vertex groups $G_{v}$, the hypotheses of Proposition 4.6(2) are satisfied and we conclude that $\mathcal{R}^{t}$ and $\mathcal{R}^{\prime t}$ are the identity on all edge groups, and that there are elements $\gamma(v) \in G_{v}$ and $\beta(e), \beta^{\prime}(e) \in G_{e}$ such that $\left(R^{t}\right)_{v}=\left(\mathcal{R}^{\prime t}\right)_{v}=a d_{\gamma(v)}, \delta_{\mathcal{R}^{t}}(e)=\gamma(\tau(e)) f_{e}(\beta(e))$ and $\delta_{\mathcal{R}^{\prime t}}(e)=\gamma(\tau(e)) f_{e}\left(\beta^{\prime}(e)\right)$. It follows from Corollary 4.7 that the twistors of $\mathcal{D}$ are given by $z_{e}=\beta(\bar{e}) \beta(e)^{-1}=\beta^{\prime}(\bar{e}) \beta^{\prime}(e)^{-1}$. But our computation above shows

Hence we obtain

$$
\begin{aligned}
& \beta^{\prime}(e)=\beta(e) b(e)^{s}, \\
& \beta^{\prime}(\bar{e})=\beta(\bar{e}) R_{e}^{k}(b(e))^{s}=\beta(\bar{e}) b(e)^{-s} .
\end{aligned}
$$

$$
\beta(\bar{e}) \beta(e)^{-1}=\beta(\bar{e}) b(e)^{-s}\left(\beta(e) b(e)^{s}\right)^{-1}=\beta(\bar{e}) \beta(e)^{-1} b(e)^{-2 s},
$$

which proves $b(e)=1$, as $s \geqslant 1$.

If $e$ is exceptional and $e$ and $\bar{e}$ are in different orbits, we may assume that $e$ and $\bar{e}$ are both exceptional. Using the basic edge relations, we 'push' some of $\delta(e)$ over to $\delta(\bar{e})$ until $\delta(\bar{e})$ is equal to $\delta^{\prime}(\bar{e})$ and $\delta^{\prime}(e)=\delta(e) f_{R(e)}\left(R_{e}\left(b^{\prime}(e)\right)\right)$ for some $b^{\prime}(e) \in G_{e}$; more precisely, replace $\delta(\bar{e})$ by $\delta^{\prime}(\bar{e})=\delta(\bar{e}) f_{R(\bar{e})}\left(R_{\bar{e}}(b(\bar{e}))\right)$ and $\delta(e)$ by $\delta(e) f_{R(e)}\left(R_{\bar{e}}(b(\bar{e}))\right)$. The resulting graph-of-groups isomorphism is equal to $\mathcal{R}$ on the path group, so we have not changed the conjugacy class of $\hat{\mathcal{R}}$.

We can now repeat the same application of Proposition 4.6 and Corollary 4.7 as in the previous case and obtain that $b^{\prime}(e)=1$ in the same way.

Thus, all data for $\mathcal{R}$ and $\mathcal{R}^{\prime}$ are identical.

The following theorem is the goal of this section. It gives necessary and sufficient criteria for two roots of efficient Dehn twists to induce conjugate outer automorphisms.

Theorem 5.7. Two $t$-th roots $\mathcal{R}: \mathcal{G} \rightarrow \mathcal{G}$ and $\mathcal{R}^{\prime}: \mathcal{G}^{\prime} \rightarrow \mathcal{G}^{\prime}$ of efficient Dehn twists $D$ and $D^{\prime}$, with twistors $\left\{z_{e}\right\}$ and $\left\{z_{e}^{\prime}\right\}$, respectively, define outer automorphisms that are conjugate by some outer isomorphism $\psi: \pi_{1}(\mathcal{G}) \rightarrow \pi_{1}\left(\mathcal{G}^{\prime}\right)$ if and only if there exists

(1) a graph isomorphism $H: \Gamma(\mathcal{G}) \rightarrow \Gamma\left(\mathcal{G}^{\prime}\right)$ that satisfies $H^{-1} R^{\prime} H=R$; and

(2) for each vertex $v \in \Gamma(\mathcal{G})$, a group isomorphism $h_{v}: G_{v} \rightarrow G_{H(v)}$ satisfying

(a) $h_{v}^{-1}\left(R^{\prime t(v)}\right)_{H(v)} h_{v}=\left(R^{t(v)}\right)_{v}$ up to inner automorphism, where $t(v)$ is the order of the $R$-orbit of $v$; and

(b) $h_{v}\left(f_{e}\left(z_{e}\right)\right)$ is conjugate to $f_{H(e)}\left(z_{H(e)}^{\prime}\right)$ for all edges e terminating at $v$. 
Proof. First, we assume that $\psi^{-1} \hat{\mathcal{R}}^{\prime} \psi=\hat{\mathcal{R}}$ for some outer isomorphism $\psi: \pi_{1}(\mathcal{G}) \rightarrow$ $\pi_{1}\left(\mathcal{G}^{\prime}\right)$. Then $\psi^{-1} \hat{D}^{\prime} \psi=\psi^{-1} \hat{\mathcal{R}}^{\prime t} \psi=\hat{\mathcal{R}}^{t}=\hat{D}$, so by Theorem 4.10 there is a graph-ofgroups isomorphism $\mathcal{H}: \mathcal{G} \rightarrow \mathcal{G}^{\prime}$ with $\psi=\hat{\mathcal{H}}$ and $H_{e}\left(z_{e}\right)=z_{H(e)}$ for all edges $e$ of $\Gamma(\mathcal{G})$. We set $h_{v}=H_{v}$. Statement $(2 \mathrm{~b})$ follows immediately. Since $\mathcal{G}$ is the graph of groups of an efficient Dehn twist, $\mathcal{G}$ is free-cyclic. Since $\hat{\mathcal{H}}^{-1} \hat{\mathcal{R}}^{\prime} \hat{\mathcal{H}}=\hat{\mathcal{R}}$, part (1) of Corollary 4.9 implies that $H^{-1} R^{\prime} H=R$, which is statement (1). Now, for any integer $k$, we get $R^{k}=H^{-1}\left(R^{\prime}\right)^{k} H$, so that the order $t(v)$ is equal to $t(H(v))$ for any vertex $v$. By part (2) of Corollary 4.9, the outer isomorphisms induced by $R_{H(v)}^{\prime} H_{v}$ and $H_{R(v)} R_{v}$ are equal, so that the outer automorphisms induced by $H_{v}^{-1}\left(R^{\prime t(v)}\right)_{H(v)} H_{v}$ and $\left(R^{t(v)}\right)_{v}$ are also equal, giving statement (2a).

For the converse, we use the given data to build a graph-of-groups isomorphism $\mathcal{H}$ : $\mathcal{G} \rightarrow \mathcal{G}^{\prime}$ such that $\hat{\mathcal{H}}^{-1} \hat{\mathcal{R}}^{\prime} \hat{\mathcal{H}}$ is conjugate in $\operatorname{Out}\left(\pi_{1}(\mathcal{G})\right)$ to $\hat{\mathcal{R}}$. It follows directly from the definition of efficient Dehn twist automorphism and from [5, Lemma 5.3] that for any graph-of-groups isomorphism $\mathcal{H}$, the conjugate $\mathcal{H}^{-1} \mathcal{R}^{\prime} \mathcal{H}$ of $\mathcal{R}^{\prime}$ is also a $t$-th root of an efficient Dehn twist, with twistors $\left\{H_{e}^{-1}\left(z_{H(e)}^{\prime}\right)\right\}$. Therefore, by Proposition 5.6 it suffices to construct $\mathcal{H}$ so that $\mathcal{H}^{-1} \mathcal{R}^{\prime} \mathcal{H}$ agrees with $\mathcal{R}$ on the graph and on all vertex groups, and $H_{e}\left(z_{e}\right)=z_{H(e)}^{\prime}$ for all edges $e$.

For the graph isomorphism of $\mathcal{H}$, we take the isomorphism $H: \Gamma(\mathcal{G}) \rightarrow \Gamma\left(\mathcal{G}^{\prime}\right)$ given in (1).

In order to define $\mathcal{H}$ on the vertex groups, we first show that we may assume that condition (2a) is a strict equality instead of just an equality up to inner automorphism. Choose a preferred vertex $v$ in each vertex orbit of $R$. Condition (2a) says that $h_{v}^{-1}\left(R^{\prime t(v)}\right)_{H(v)} h_{v}=a d_{\gamma} \circ\left(R^{t(v)}\right)_{v}$ for some $\gamma \in G_{v}$. Let $v_{1}=R^{t(v)-1}(v)$. By Corollary 4.8, we may replace $R_{v_{1}}: G_{v_{1}} \rightarrow G_{v}$ by $a d_{\gamma} \circ R_{v_{1}}$ and $\delta(e)$ by $\gamma \delta(e)$ for all edges $e$ terminating at $v_{1}$ without changing the outer automorphism $\hat{\mathcal{R}}$. After this change, we have

$$
h_{v}^{-1}\left(R^{\prime t(v)}\right)_{H(v)} h_{v}=\left(R^{t(v)}\right)_{v} .
$$

Our modification has not changed the fact that $\mathcal{R}^{t}$ represents an efficient Dehn twist based on $\mathcal{G}$, and the twistors are unchanged. Furthermore, the hypothesis (2b) is still valid.

Now let $u$ be any vertex. Then $u$ is in the orbit of some preferred vertex $v$, say $u=R^{k}(v)$ for some $0 \leqslant k \leqslant t(v)-1$. We define the vertex group map $H_{u}$ for the desired isomorphism $\mathcal{H}$ by $H_{u}=\left(R^{\prime k}\right)_{H(v)} h_{v}\left(R^{-k}\right)_{u}$, giving $H_{R(u)}^{-1} R_{H(u)}^{\prime} H_{u}=R_{u}$.

It remains to show that there exist edge homomorphisms $H_{e}$ and correction terms $\delta(e)$ satisfying the conditions for $\mathcal{H}$ to be a graph-of-groups isomorphism, and that $H_{e}\left(z_{e}\right)=$ $z_{H(e)}^{\prime}$ for all edges $e$.

Let $e$ be any edge of $\Gamma$, with terminal vertex $v$. Since $\mathcal{R}$ and $\mathcal{R}^{\prime}$ are graph-of-groups isomorphisms, the vertex maps commute with the edge injections up to conjugacy; thus, condition (2b) implies that $H_{v} f_{e}\left(z_{e}\right)$ is conjugate to $f_{H(e)} z_{H(e)}^{\prime}$. Since these two elements are conjugate in $G_{H(v)}$, the maximal cyclic subgroups that contain them are conjugate. Recall that for efficient Dehn twists, edge groups are always mapped to maximal cyclic 
subgroups in the adjacent vertex groups; we conclude that $H_{v} f_{e}\left(G_{e}\right)=a d_{x} f_{H(e)}\left(G_{H(e)}\right)$ for some element $x \in G_{H(v)}$. Choose generators $y$ for $G_{e}$ and $y^{\prime}$ for $G_{H(e)}$ that map to the same element of $G_{H(v)}$, and define $H_{e}$ by sending $y$ to $y^{\prime}$. We now check that setting $\delta(e)=x$ satisfies the conditions for $\mathcal{H}$ to be a graph-of-groups isomorphism. Finally, condition (2b) implies that $H_{e}\left(z_{e}\right)=z_{H(e)}^{\prime}$.

\subsection{Solving the conjugacy problem}

Algorithm 5.8. We now describe the algorithm for deciding whether two given roots of Dehn twist automorphisms are conjugate. Here, we assume that the given data are, for $i=1,2$ :

(1) a graph of groups $\mathcal{G}_{i}^{\prime}$;

(2) an outer automorphism $\phi_{i} \in \operatorname{Out}\left(\pi_{1}\left(\mathcal{G}_{i}^{\prime}\right)\right)$; and

(3) an integer $t \geqslant 0$ and a Dehn twist $D_{i}^{\prime}$ based on $\mathcal{G}_{i}^{\prime}$, such that $\phi_{i}^{t}=\hat{D}_{i}^{\prime}$.

(One should think of $t$ as a common multiple of exponents $t_{i}$, such that the $\phi_{i}^{t_{i}}$ are Dehn twist automorphisms.)

We then proceed as follows.

(1) Find the efficient Dehn twist $D_{i}$ based on a graph of groups $\mathcal{G}_{i}$ with twistors $\left\{z_{e}\right\}$, such that $\hat{D}_{i}$ is conjugate to $\hat{D}_{i}^{\prime}$. How this can be done in finitely many steps is explained in $[\mathbf{5}, \S 8]$.

(2) Find a graph-of-groups automorphism $\mathcal{R}_{i}: \mathcal{G}_{i} \rightarrow \mathcal{G}_{i}$ that realizes the root of $\hat{D}_{i}$ conjugate to $\phi_{i}$. Such $\mathcal{R}_{i}$ exists, by Proposition 5.3, and can be found in finitely many steps since the set of all graph-of-groups automorphisms of $\mathcal{G}_{i}$ is enumerable. (In practice, one can find $\mathcal{R}_{i}$ more efficiently using the starting data and the conjugacy between $\mathcal{G}_{i}$ and $\mathcal{G}_{i}^{\prime}$.)

(3) List all graph isomorphisms $H: \Gamma\left(\mathcal{G}_{1}\right) \rightarrow \Gamma\left(\mathcal{G}_{2}\right)$ that satisfy $H^{-1} R_{2} H=R_{1}$.

(4) For each $H$ found in step (3) and for every vertex $v$ of $\Gamma\left(\mathcal{G}_{1}\right)$, check whether there exists an isomorphism $h_{v}: G_{v} \rightarrow G_{H(v)}$ that satisfies:

(a) $h_{v}^{-1}\left(R_{2}^{t(v)}\right)_{H(v)} h_{v}=\left(R_{1}^{t(v)}\right)_{v}$ up to inner automorphisms, where $t(v)$ denotes the cardinality of the $R_{1}$-orbit of $v$; and

(b) $h_{v}\left(f_{e}\left(z_{e}\right)\right)$ is conjugate to $f_{H(e)}\left(z_{H(e)}\right)$ for all edges $e$ of $\Gamma\left(\mathcal{G}_{1}\right)$ with $\tau(e)=v$.

Since $\left(R_{1}^{t(v)}\right)_{v}$ and $\left(R_{2}^{t(v)}\right)_{H(v)}$ are finite-order (equal to $t / t(v)$ ) automorphisms of free groups, this step may be done by the equivariant Whitehead algorithm derived in $\S 2$ of this paper.

It follows directly from Theorem 5.7 that a graph-of-groups isomorphism $\mathcal{H}$ with the properties (4a) and (4b) above exists if and only if $\phi_{1}$ and $\phi_{2}$ are conjugate. Hence, the above algorithm is a solution of the conjugacy problem for roots of Dehn twist automorphisms of finitely generated free groups. 
Acknowledgements. S.K. was partly supported by a grant from the Science Fund of Serbia through Matematički Institut Belgrade. K.V. was partly supported by NSF grant DMS-9971607.

\section{References}

1. H. BAss, Covering theory for graphs of groups, J. Pure Appl. Algebra 89 (1993), 3-47.

2. M. Bestvina And M. Handel, Train tracks and automorphisms of free groups, Ann. Math. 135 (1992), 1-51.

3. M. Bestvina, M. Feighn and M. Handel, The Tits alternative for $\operatorname{Out}\left(F_{n}\right)$, I and II, Preprint (1997).

4. M. M. Cohen And M. Lustig, Very small group actions on R-trees and Dehn twist automorphisms, Topology 34 (1995), 575-617.

5. M. M. Cohen And M. Lustig, The conjugacy problem for Dehn twist automorphisms of free groups, Commentarii Math. Helv. 74(2) (1999), 179-200.

6. M. Culler, Finite groups of outer automorphisms of a free group, in Contributions to group theory, pp. 197-207, Contemporary Mathematics, vol. 33 (American Mathematical Society, Providence, RI, 1984).

7. S. KALAJDŽIEvSKi, Automorphism group of a free group: centralizers and stabilizers, $J$. Algebra 150 (1992), 435-502.

8. S. KRstić, Actions of finite groups on graphs and related automorphisms of free groups, J. Algebra 124 (1989), 119-138.

9. S. KRSTIĆ, Finitely generated virtually free groups have finitely presented automorphism group, Proc. Lond. Math. Soc. 64 (1992), 49-69.

10. S. Krstić And K. Vogtmann, Equivariant outer space and automorphisms of free-byfinite groups, Comment. Math. Helv. 68 (1993), 216-262.

11. J. Los, On the conjugacy problem for automorphisms of free groups, Topology 35 (1996), 779-808.

12. M. Lustig, Prime factorization and conjugacy problem in $\operatorname{Out}\left(F_{n}\right)$, Survey preprint (July 1994).

13. M. LUSTIG, Structure and conjugacy for automorphisms of free groups, Preprint (April, 1999).

14. Z. SElA, The isomorphism problem for hyperbolic groups, I, Ann. Math. 141 (1995), $217-283$.

15. J. H. C. Whitehead, On equivalent sets of elements in a free group, Ann. Math. 37 (1936), 782-800.

16. B. Zimmermann, Über Homöomorphismen $n$-dimensionaler Henkelkörper und endliche Erweiterungen von Schottky-Gruppen (On homeomorphisms of $n$-dimensional handlebodies and on finite extensions of Schottky groups), Comment. Math. Helv. 56 (1981), 474-486. 